\title{
Dold spaces in homotopy theory
}

\author{
EUGENIA SCHWAMBERGER \\ RAINER M VOGT
}

\begin{abstract}
We study a class of spaces whose importance in homotopy theory was first highlighted by work of Dold in the 1960s, and that we accordingly call Dold spaces. These are the spaces that possess a partition of unity supported in sets that are contractible to a point within the ambient space. Dold spaces form a broader class than spaces homotopy equivalent to $\mathrm{CW}$ complexes, but share the feature that a number of well known weak equivalences are genuine ones if Dold spaces are involved. In this paper we give a first systematic investigation of Dold spaces. After listing their elementary properties, we study homotopy pullbacks involving Dold spaces and simplicial objects in the category of Dold spaces. In particular, we show that the homotopy colimit of a diagram of Dold spaces is a Dold space and that the topological realization functor preserves fibration sequences if the base is a path-connected Dold space in each dimension. It follows that the loop space functor commutes with realization up to homotopy for Dold spaces. Finally, we give simple conditions which assure that free algebras over a topological operad are Dold spaces.
\end{abstract}

55P99; 55P35, 55P48, 55U10

\section{Introduction}

A Dold space is a topological space $X$ which admits a partition of unity by functions $f_{\alpha}$ whose supports form a locally finite cover of $X$ by sets $S_{\alpha}$ for which the inclusion maps $S_{\alpha} \hookrightarrow X$ are nullhomotopic. Dold spaces include CW complexes and, more generally, all paracompact locally contractible spaces as well as spaces homotopy equivalent to such spaces since a space homotopy equivalent to a Dold space is a Dold space. Other examples are the classifying spaces of wellpointed topological monoids (see Corollary 5.2), arbitrary nonreduced suspensions and, more generally, the join $X * Y$ of any two spaces. The suspension of a compact space whose homology is not finitely generated does not have the homotopy type of a CW complex (as shown in Example 2.6), so Dold spaces are more general than the standard category of spaces homotopy equivalent to $\mathrm{CW}$ complexes.

The purpose of this paper is to give the first systematic account of Dold spaces. We start by recalling what is known about them so far. 
A Dold was the first who realized the importance of Dold spaces in homotopy theory: recall that a map $p: E \rightarrow B$ is a homotopy fibration or $h$-fibration, for short, if it is fiberwise homotopy equivalent to a fibration $q: \widehat{E} \rightarrow B$. In 1963 Dold [5, Theorem 6.3] proved:

Theorem 1.1 Given a commutative diagram

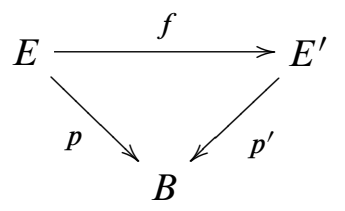

such that $p$ and $p^{\prime}$ are $h$-fibrations and $B$ is a Dold space then $f$ is a fiberwise homotopy equivalence if and only if its restriction to every fiber is a homotopy equivalence.

By explicitly constructing an appropriate partition of unity Dold [5, Proposition 6.7] also proved:

Proposition 1.2 Each CW complex is a Dold space.

In 1970 and 1971 D Puppe used Dold's result to show that some important weak homotopy equivalences are genuine ones. Let $k$ Top* denote the category of based $k$-spaces, ie compactly generated not necessarily weak Hausdorff spaces. For $X$ in $k$ Top* let $J X$ denote the James construction on $X$ in $k$ Top*, ie the based free topological monoid on $X$. In [4, Section 17] and [14, Section 3] Puppe gave two different proofs of the following:

Theorem 1.3 If $X$ is an $h$-wellpointed, path-connected Dold space, then $J X \simeq$ $\Omega \Sigma X$.

For a definition of $h$-wellpointed, see Definition 3.11.

In 1981 Puppe's student $\mathrm{H}$ Meiwes proved a related result. For $X$ in $k$ Top* let $\mathcal{C}_{n}^{*}(X)$ denote the based free algebra over the operad $\mathscr{C}_{n}$ of little $n$-cubes. P May [10] constructed a weak equivalence $\mathcal{C}_{n}^{*}(X) \rightarrow \Omega^{n} \Sigma^{n} X$ for $X$ path-connected. In his thesis [12], Meiwes proved:

Theorem 1.4 If $X$ is an $h$-wellpointed, path-connected Dold space, then May's map $\mathcal{C}_{n}^{*}(X) \rightarrow \Omega^{n} \Sigma^{n} X$ is a genuine homotopy equivalence. 
In the context of these theorems we share D Puppe's point of view [14]: "Frequently a weak homotopy equivalence is considered as good as a genuine one, because for spaces having the homotopy type of a CW-complex there is no difference and most interesting spaces in algebraic topology are of that kind. I am not going to argue against this because I agree with it, but I do think that the methods by which we establish the genuine homotopy equivalences give some new insight into homotopy theory."

Indeed, if you try to construct genuine homotopy equivalences between spaces which are not necessarily of the homotopy type of CW-complexes you do not have the algebraic side of homotopy theory at your disposal. So these constructions have a different, more geometric flavor. Algebraic tools like homotopy or homology groups give you a weak homotopy equivalence. But a weak homotopy equivalence between Dold spaces need not be a genuine one, because otherwise Dold spaces would be equivalent to $\mathrm{CW}$ complexes by taking $\mathrm{CW}$ approximations of them.

Another result illustrating this is G Allaud's delooping of homotopy equivalences of 1972 [1]. A based map $f: X \rightarrow Y$ of path-connected spaces such that $\Omega f: \Omega X \rightarrow \Omega Y$ is a homotopy equivalence is a weak homotopy equivalence, but it need not be a genuine one: the loop space of the Warsaw circle is contractible, but the Warsaw circle is not. Allaud proved:

Proposition 1.5 Let $f: X \rightarrow Y$ be a based map of path-connected Dold spaces such that $\Omega f: \Omega X \rightarrow \Omega Y$ is a homotopy equivalence. Then $f$ is a homotopy equivalence.

Results about properties of Dold spaces are fairly scarce in the literature. Meiwes and Puppe proved some technical lemmas, which replaced quasi-fibrations by maps closer to a fibration and which involved Dold spaces. They needed these results for their proofs of Theorems 1.4 and 1.3. But one can find only few properties of Dold spaces in print.

In $[4 ; 14]$, Puppe just listed the properties he needed in the context of Theorem 1.3. In $[4,(12.7)$ and (17.3)] he proved:

Proposition 1.6 (1) Let $X$ be a Dold space with a homotopy associative multiplication $\mu: X \times X \rightarrow X$ with a homotopy unit $e$. If $\mu$ induces a group structure on $\pi_{0}(X)$, then $\mu$ has a homotopy inverse inv: $X \rightarrow X$.

(2) If $X$ dominates $Y$ and $X$ is a Dold space then so is $Y$. 
In [14] you find:

Lemma 1.7 (1) The product of two Dold spaces is a Dold space.

(2) The (nonreduced) mapping cone of a map $f: X \rightarrow Y$ into a Dold space $Y$ is a Dold space. The (nonreduced) suspension of any space is a Dold space.

(3) The same holds for the reduced versions provided all spaces are $h$-wellpointed.

(4) If $X$ and $Y$ are $h$-wellpointed Dold spaces, then $X \vee Y$ and $X \wedge Y$ are Dold spaces.

(5) The colimit of a sequence of h-cofibrations (for a definition see Definition 3.5) of Dold spaces is a Dold space.

For the proof of Theorem 1.4 Meiwes also needed [12, Satz 3.4]:

Proposition 1.8 The $k$-fold symmetric product $\mathrm{SP}_{k}(X)=X^{k} / \Sigma_{k}$ of a Dold space $X$ is a Dold space.

Let $\triangle$ denote the category of finite ordered sets $[n]=\{0<1<\cdots<n\}$ and order preserving maps. A simplicial space is a functor $X_{\bullet}: \triangle^{\text {op }} \rightarrow$ Top, $[n] \mapsto X_{n}$. Let $\nabla: \triangle \rightarrow$ Top be the standard simplex functor mapping $[n]$ to the standard $n-\operatorname{simplex} \Delta^{n}$. The (thin) topological realization $\left|X_{\bullet}\right|$ is defined as

$$
\left(\coprod_{n \geq 0} X_{n} \times \Delta^{n}\right) / \sim
$$

with the relations $\left(X_{\bullet}(\alpha)(x), t\right) \sim(x, \nabla(\alpha)(t))$ for $\alpha \in \Delta([k],[l]), x \in X_{l}, t \in \Delta^{k}$. If we restrict the relations to injective morphisms $\alpha$ in $\triangle$ we obtain the fat realization $\left\|X_{\bullet}\right\|$.

An element $x \in X_{n}$ is called degenerate if it is in the image of some $X_{\bullet}(\alpha)$ with $\alpha \neq$ id surjective. We call $X_{\bullet}$ proper if the inclusions $s X_{n} \subset X_{n}$ of the subspaces of degenerate elements are cofibrations for all $n$.

Remark 1.9 Apparently D Puppe knew a lot more about Dold spaces than has appeared in print:

(1) In [16] G Segal attributed the following result to Puppe: The topological realization of a proper simplicial space is a Dold space if the space of 0 -simplices is contractible. (No proof is given.)

(2) Let $X_{\bullet}$ denote a based simplicial space, ie a functor from $\triangle^{\text {op }}$ to Top* , and let $\Omega Y$ denote the loop space of $Y$. In discussions with the second author, Puppe sketched a proof of the fact that $\left\|\Omega X_{\bullet}\right\|$ is homotopy equivalent to $\Omega\left\|X_{\bullet}\right\|$ if each $X_{n}$ is a path-connected Dold space. 
Recently Dold spaces entered into work of J Smrekar who proved that the space of functions between two $\mathrm{CW}$ complexes is of the homotopy type of a CW complex if and only if it is a Dold space [17, Theorem 2.2.1]. The proof uses Allaud's Proposition 1.5 above; no further interesting properties of Dold spaces are mentioned. Smrekar also coined the name Dold space.

Overview of the results of this paper In Section 2 we will recall the definition of Dold spaces and some facts about covers admitting a partition of unity. In Section 3 we will list a number of elementary facts about Dold spaces including proofs of Proposition 1.6 and the statements of Lemma 1.7. Statement (1) of Lemma 1.7 is fairly obvious. We extend it to:

Proposition 3.10 The total space of a fibration is a Dold space if the base and the fibers are Dold spaces.

Statements (2) to (5) of Lemma 1.7 are consequences of:

Proposition 3.4 and Proposition 3.13 The double mapping cylinder of $f: A \rightarrow X$ and $g: A \rightarrow Y$ is a Dold space if $X$ and $Y$ are Dold spaces. If $f$ and $g$ are based maps and $A, X$, and $Y$ are $h$-wellpointed the same holds for the reduced double mapping cylinder.

In Corollary 3.9 we give an alternative proof of Proposition 1.2.

Good tools for proving results like Theorem 1.3 are homotopy pullbacks and homotopy pushouts. Homotopy pullbacks of Dold spaces have particularly nice properties. With the exception of one statement, The cube theorems 4.8, we will prove them in Section 4. We demonstrate their usefulness by giving comparatively short proofs of Proposition 1.5 and an extension of Theorem 1.3.

Homotopy pullbacks and homotopy pushouts will also be used in the study of simplicial Dold spaces in Section 5. We first prove a useful extension of Remark 1.9 (1) due to D Puppe and communicated to us by Meiwes [13]:

Proposition 5.1 If $X_{\bullet}$ is a simplicial space such that $X_{0}$ is a Dold space, then $\left\|X_{\bullet}\right\|$ is a Dold space. If, in addition, $X_{\bullet}$ is proper, then $\left|X_{\bullet}\right|$ is a Dold space.

From this we deduce: 
Proposition 5.3 and Proposition 5.4 The homotopy colimit of a diagram of Dold spaces is a Dold space. The reduced homotopy colimit of a diagram of $h$-wellpointed Dold spaces is a Dold space.

We then give a characterization of Dold spaces. For a based space $\left(X, x_{0}\right)$ let $\operatorname{Sing}^{0} X$ denote the simplicial space whose $n$-th space $\operatorname{Sing}_{n}^{0} X$ is the space of maps $\left(\Delta^{n}, \Delta_{0}^{n}\right) \rightarrow$ $\left(X, x_{0}\right)$, where $\Delta_{0}^{n}$ is the 0 -skeleton of the $n$-simplex $\Delta^{n}$. The evaluation maps $\operatorname{Sing}_{n}^{0} X \times \Delta^{n} \rightarrow X$ induce a map

$$
\text { ev: }\left\|\operatorname{Sing}_{\bullet}^{0} X\right\| \rightarrow X .
$$

Proposition 5.6 A path-connected based space $X$ is a Dold space if and only if ev: $\left\|\operatorname{Sing}_{\bullet}^{0} X\right\| \rightarrow X$ is a homotopy equivalence.

We use this to show:

Proposition 5.17 Given a commutative diagram of based simplicial spaces

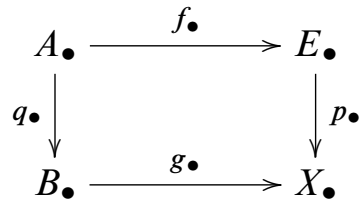

which is a homotopy pullback for each $[n] \in \mathrm{ob} \triangle$, then

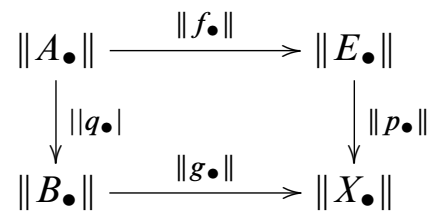

is a homotopy pullback, provided each $B_{n}$ and each $X_{n}$ is a path-connected Dold space.

Remark 1.9 (2) is an immediate consequence.

In Section 6 we apply these results to algebras over an operad $\mathscr{P}$ in the categories $k$ Top and $k$ Top* of $k$-spaces respectively based $k$-spaces. We call $\mathscr{P}$ reduced if $\mathscr{P}(0)$ consists of a single element. If $X$ is a $\mathscr{P}$-space and $\mathscr{P}$ is reduced, the single element of $\mathscr{P}(0)$ determines a basepoint in $X$. Let $\mathcal{P}$ Top be the category of $\mathscr{P}$-spaces. The forgetful functors

$$
U: \mathcal{P} \text { Top } \rightarrow k \text { Top }
$$


and, if $\mathscr{P}$ is reduced,

$$
U^{*}: \mathcal{P} \text { Top } \rightarrow k \text { Top }^{*}
$$

have left adjoints

$$
\mathcal{P}: k \text { Top } \rightarrow \mathcal{P} \text { Top, } \quad \text { respectively, } \quad \mathcal{P}^{*}: k \text { Top }^{*} \rightarrow \mathcal{P} \text { Top } .
$$

We show:

Proposition 6.3 If $\mathscr{P}$ is an operad such that each $\mathscr{P}(n) / \Sigma_{n}$ is a Dold space and $X \in k$ Top is a path-connected Dold space, then $\mathcal{P}(X)$ is a Dold space.

Proposition 6.4 If $X \in k$ Top* is an $h$-wellpointed path-connected Dold space, then $\mathcal{P}^{*}(X)$ is a Dold space for each reduced operad $\mathscr{P}$.

Proposition 1.8 is an easy consequence of Proposition 6.3.

We close with a remark about function spaces. We had an email exchange with J Smrekar about function space properties of Dold spaces. They turned out to be so restrictive that we did not include them in this paper. In fact, the category of Dold spaces is rather badly behaved with respect to function spaces. In particular, loop spaces of Dold spaces need not be Dold spaces (as shown in Example 5.15).

Prerequisites The reader is supposed to be familiar with the basic properties of fibrations, cofibrations and simplicial spaces. An excellent reference for fibrations, cofibrations, $h$-fibrations and $h$-cofibrations is tom Dieck, Kamps and Puppe [4]. For basic properties of simplicial spaces and their realizations we recommend May [10, Sections 11 and 12; 11, Appendix]. Boardman and Vogt [2, Appendix] may also be helpful. For Section 6 some knowledge of operads and their algebras is required, but Sections 1 and 2 of [10] will suffice. We will rely on Theorem 1.1 and the first cube theorem 4.8, but we will not prove them. Neither will we prove the second cube theorem 4.8, which we include because it contains a Dold space version. Proofs of the first cube theorem and the first part of the second cube theorem as well as more details about homotopy pushouts and pullbacks can be found in Mather [9].

Acknowledgments We thank A Hatcher for an email exchange about Example 2.6 and J Smrekar for an email exchange about function space properties of Dold spaces and suggesting the name "Dold space". We are indebted to E Floyd and W Floyd for contributions to Section 4. Most of all we are indebted to the referee who generalized Hatcher's Example 2.6 and made a large number of very helpful suggestions about the organization of the paper and the explicit formulations of parts of the abstract and the introduction. 


\section{Dold covers}

In this section we recall the basic definitions and list results related to coverings. Let $\left\{a_{j} ; j \in J\right\}$ denote a set of elements of $\mathbb{R}_{+}=\{x \in \mathbb{R} ; x \geq 0\}$. We define

$$
\sum_{j \in J} a_{j}=\sup \left\{\sum_{j \in E} a_{j} ; E \subset J \text { finite }\right\} .
$$

Definition 2.1 A partition of unity on a space $X$ is a set of maps $\left\{f_{j}: X \rightarrow[0,1] ; j \in J\right\}$ such that

$$
\sum_{j \in J} f_{j}(x)=1 \quad \text { for all } x \in X
$$

Definition 2.2 Let $X$ be a space. A subset $A \subset X$ is called ambiently contractible if the inclusion $A \rightarrow X$ is nullhomotopic.

Definition 2.3 Let $\mathcal{U}=\left\{U_{\alpha} ; \alpha \in A\right\}$ be a cover of $X$.

(1) $\mathcal{U}$ is called locally finite if each $x \in X$ has a neighborhood $V$ such that $V \cap U_{\alpha} \neq$ $\varnothing$ for only finitely many $\alpha \in A$.

(2) A numeration of $\mathcal{U}$ is a partition of unity $\left\{f_{\alpha} ; \alpha \in A\right\}$ on $X$ such that the set $\left\{\operatorname{Supp}\left(f_{\alpha}\right) ; \alpha \in A\right\}$ is locally finite and $\operatorname{Supp}\left(f_{\alpha}\right) \subset U_{\alpha}$ for all $\alpha \in A$. (Recall, the support $\operatorname{Supp}(f)$ of a map $f: X \rightarrow I$ is the closure of $\left.\left.f^{-1}(] 0,1\right]\right)$.) If $\mathcal{U}$ admits a numeration, it is called a numerable cover.

(3) $\mathcal{U}$ is called an ambiently contractible cover if each $U_{\alpha}$ is ambiently contractible.

(4) $\mathcal{U}$ is called a Dold cover if it is numerable and ambiently contractible.

Definition 2.4 A space $X$ is called ambiently locally contractible if it has an ambiently contractible open cover. We call $X$ a Dold space if it has a Dold cover.

Remark In the literature the term "weakly contractible" or "semilocally contractible" is used for what we call "ambiently contractible". In [4] D Puppe uses the term "numerierbare nullhomotope Überdeckung" (numerable nullhomotopic cover) for a Dold cover and in [14] the term "numerably contractible space" for a Dold space. For Allaud a Dold space is a space which is "locally contractible in the large" [1] and for Meiwes a space which is "numerierbar lokal zusammenziehbar" (numerably locally contractible) [12]. 
Examples 2.5 (1) Each contractible space is a Dold space.

(2) The unreduced suspension of an arbitrary space is a Dold space.

(3) More generally, the join of any two spaces is a Dold space.

(4) Each paracompact ambiently locally contractible space is a Dold space.

(5) By [7, Theorem II.3] each paracompact locally equiconnected space is a Dold space.

(6) The product of two Dold spaces is a Dold space.

(7) Each CW-complex is a Dold space (see Corollary 3.9).

(8) The classifying space of an $h$-wellpointed topological monoid is a Dold space (see Corollary 5.2).

We will show in Corollary 3.3 that a space of the homotopy type of a Dold space is a Dold space. So the standard category of spaces homotopy equivalent to CW complexes is a subcategory of the category of Dold spaces. The following example shows that the latter is larger than the former.

Example 2.6 (1) Let $X$ be the unreduced suspension of a compact space $Y$ with $H_{*}(Y)$ not finitely generated (eg take $Y=\left\{\frac{1}{n} ; n \in \mathbb{N}\right\} \cup\{0\} \subset \mathbb{R}$ ). Then $X$ is a Dold space which is not homotopy equivalent to a $\mathrm{CW}$ complex.

(2) A weak homotopy equivalence between Dold spaces need not be a genuine one.

Proof (1) Let $f: X \rightarrow Z$ be any map into a CW complex $Z$. Since $X$ is compact $f$ factors through a finite subcomplex $A \subset Z$, and $f_{*}: H_{*}(X) \rightarrow H_{*}(Z)$ factors through $H_{*}(A)$. Since $H_{*}(X)$ is not finitely generated but $H_{*}(A)$ is, the map $f_{*}$ cannot be injective and hence $f$ cannot be a homotopy equivalence.

(2) If $q: \bar{X} \rightarrow X$ is a CW approximation of $X$, then $q$ is a weak homotopy equivalence, but not a genuine one.

We will make use of the following results.

Lemma 2.7 [3, page 347] If $\left\{f_{j}, j \in J\right\}$ is a partition of unity on $X$, then $\left.\left.\left\{f_{j}^{-1}(] 0,1\right]\right) ; j \in J\right\}$ is a numerable cover of $X$.

Lemma 2.8 [3, page 349] Let $\left\{f_{j}: X \rightarrow \mathbb{R}_{+} ; j \in J\right\}$ be a set of maps such that $\mathcal{U}=\left\{f^{-1}(] 0, \infty[) ; j \in J\right\}$ is a locally finite cover of $X$, then $\mathcal{U}$ is a numerable cover. 
Corollary 2.9 The following are equivalent:

(1) $X$ is a Dold space.

(2) $X$ has a partition of unity $\left\{f_{j} ; j \in J\right\}$ such that $\left.\left.\left\{f_{j}^{-1}(] 0,1\right]\right) ; j \in J\right\}$ is an ambiently contractible open cover of $X$.

(3) there is a set of maps $\left\{f_{j}: X \rightarrow \mathbb{R}_{+} ; j \in J\right\}$ such that $\left\{f_{j}^{-1}(] 0, \infty[) ; j \in J\right\}$ is a locally finite ambiently contractible cover of $X$.

Proposition 2.10 Let $\mathcal{U}=\left\{U_{\alpha} ; \alpha \in A\right\}$ be a cover of $X$ by Dold spaces. Suppose that $\mathcal{U}$ has a numerable refinement $\mathcal{V}=\left\{V_{j} ; j \in J\right\}$, ie a numerable cover of $X$ such that each $V_{j}$ is contained in some $U_{\alpha}$. Then $X$ is a Dold space.

Proof Let $\left\{f_{j} ; j \in J\right\}$ be a numeration of $\mathcal{V}$. Since each $U_{\alpha}$ is a Dold space there are partitions of unity

$$
\left\{g_{\alpha, k}: U_{\alpha} \rightarrow I ; k \in K_{\alpha}\right\}
$$

such that $\left\{\operatorname{Supp}\left(g_{\alpha, k}\right) ; k \in K_{\alpha}\right\}$ is locally finite and $\operatorname{Supp}\left(g_{\alpha, k}\right)$ is contractible in $U_{\alpha}$ and hence in $X$ for all $k \in K_{\alpha}$.

Choose a function $\beta: J \rightarrow A$ such that $V_{j} \subset U_{\beta(j)}$. For $k \in K_{\beta(j)}$ define $f_{j, k}: X \rightarrow$ $[0,1]$ by

$$
f_{j, k}(x)= \begin{cases}f_{j}(x) \cdot g_{\beta(j), k}(x) & \text { for } x \in \operatorname{Supp}\left(f_{j}\right), \\ 0 & \text { for } \left.\left.x \in X \backslash f_{j}^{-1}(] 0,1\right]\right) .\end{cases}
$$

Then $f_{j, k}$ is well-defined and continuous, because $\operatorname{Supp}\left(f_{j}\right)$ and $\left.\left.X \backslash f_{j}^{-1}(] 0,1\right]\right)$ are closed in $X$. The collection $\left\{f_{j, k} ; j \in J, k \in K_{\beta(j)}\right\}$ is a partition of unity and

$$
\left.\left.\left.\left.\left.\left.f_{j, k}^{-1}(] 0,1\right]\right)=f_{j}^{-1}(] 0,1\right]\right) \cap g_{\beta(j), k}(] 0,1\right]\right) \subset \operatorname{Supp}\left(g_{\beta(j), k}\right) .
$$

Hence $\left.\left.f_{j, k}^{-1}(] 0,1\right]\right)$ is contractible in $X$. Now apply Corollary 2.9.

The numeration condition on the cover $\mathcal{U}$ in Proposition 2.10 is essential as the following example shows.

Example 2.11 Let $X \subset \mathbb{R}^{2}$ be the cone on $M=\{(0,0)\} \cup\left\{\left(\frac{1}{n}, 0\right) ; n \in \mathbb{N}\right\}$ with cone point $(0,1)$. Then $X$ is a Dold space. Now let

$$
Y=(X \sqcup X) /(0,0) \sim(0,0) .
$$

The two copies of $X$ form a closed cover of $Y$, but $Y$ is not a Dold space, because no open neighborhood of $(0,0)$ is contractible in $Y$. 


\section{Elementary properties}

Suppose $U \subset X$ is ambiently contractible in $X$ to a point $x_{0}$, then $U$ must lie in the path-component of $x_{0}$. We obtain:

Proposition $3.1 \quad$ (1) $X$ is a Dold space if and only if its path-components are open and Dold spaces.

(2) If $X=\bigsqcup_{j \in J} X_{j}$, then $X$ is a Dold space if and only if each summand $X_{j}$ is a Dold space.

This observation allows us to restrict our attention to path-connected Dold spaces.

Proposition 3.2 A space $Y$ dominated by a Dold space $X$ is itself a Dold space.

Proof [4, page 235] Let $\left\{V_{\lambda} ; \lambda \in \Lambda\right\}$ be a Dold cover of $X$, and $f: X \rightarrow Y$ and $g: Y \rightarrow X$ be maps such that $f \circ g \simeq \operatorname{id}_{Y}$. Then $\left\{g^{-1}\left(V_{\lambda}\right) ; \lambda \in \Lambda\right\}$ is a numerable cover of $Y$ and each $g^{-1}\left(V_{\lambda}\right)$ is contractible in $Y$ because

$$
g^{-1}\left(V_{\lambda}\right) \stackrel{g}{\longrightarrow} V_{\lambda} \subset X \stackrel{f}{\longrightarrow} Y
$$

is nullhomotopic and homotopic to the inclusion $g^{-1}\left(V_{\lambda}\right) \subset Y$.

Corollary 3.3 If $X$ and $Y$ are homotopy equivalent then $X$ is a Dold space if and only if $Y$ is a Dold space.

Proposition 3.4 Given a diagram

$$
X \stackrel{f}{\longleftarrow} A \stackrel{g}{\longrightarrow} Y
$$

with $X$ and $Y$ Dold spaces, then the double mapping cylinder $\widehat{M}(f, g)$ is a Dold space.

Proof $\widehat{M}(f, g)=(X \sqcup A \times[0,1] \sqcup Y) / \sim$ with $(a, 0) \sim f(a)$ and $(a, 1) \sim g(a)$. Let $U$ be the image of $X \sqcup A \times[0,1[$ in $\widehat{M}(f, g)$ and $V$ the image of $A \times] 0,1] \sqcup Y)$. Then $\{U, V\}$ is a numerable cover of $\widehat{M}(f, g)$ by Dold spaces, because $U \simeq X$ and $V \simeq Y$. Hence $\widehat{M}(f, g)$ is a Dold space by Proposition 2.10 . 
Definition 3.5 A map $f: A \rightarrow X$ is called an $h$-cofibration if there is a commutative triangle

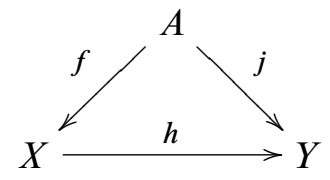

with $j$ a cofibration and $h$ a homotopy equivalence under $A$. Dually, an $h$-fibration is a map $f: A \rightarrow X$ which is homotopy equivalent over $X$ to a fibration $E \rightarrow X$.

\section{Corollary 3.6 Let}

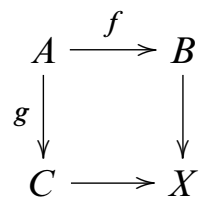

be a pushout square with $f$ an $h$-cofibration and $B$ and $C$ Dold spaces. Then $X$ is a Dold space.

Proof Since $f$ is an $h$-cofibration, the canonical map $\widehat{M}(f, g) \rightarrow X$ is a homotopy equivalence.

Corollary 3.7 (1) For any map $f: A \rightarrow X$ into a Dold space $X$, the unreduced mapping cone is a Dold space.

(2) If $f: A \rightarrow X$ is an $h$-cofibration and $X$ a Dold space, then $X / f(A)$ is a Dold space.

Proposition 3.8 Let $X_{0} \stackrel{f_{0}}{\longrightarrow} X_{1} \stackrel{f_{1}}{\longrightarrow} X_{2} \stackrel{f_{2}}{\longrightarrow} \cdots$ be a sequence of maps of Dold spaces. Then

(1) the mapping telescope $T X=\left(\bigsqcup_{n \geq 0} X_{n} \times I\right) / \sim$ with $(x, 1) \in X_{n} \times I$ related to $\left(f_{n}(x), 0\right) \in X_{n+1} \times I$ is a Dold space.

(2) if each $f_{i}$ is an h-cofibration, colim $X_{n}$ is a Dold space.

Proof $T X$ is the double mapping cylinder of

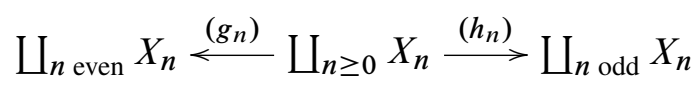

with

$$
g_{n}(x)=\left\{\begin{array}{ll}
x & n \text { even, } \\
f_{n}(x) & n \text { odd, }
\end{array} \quad h_{n}(x)= \begin{cases}f_{n}(x) & n \text { even }, \\
x & n \text { odd }\end{cases}\right.
$$

If all the $f_{n}$ are h-cofibrations, the canonical map $T X \rightarrow \operatorname{colim} X_{n}$ is a homotopy equivalence. 
Corollary 3.9 [5, Proposition 6.7] Each CW-complex $X$ and hence each space of the homotopy type of a $\mathrm{CW}$-complex is a Dold space.

Proof Let $X^{(n)}$ denote the $n$-skeleton of $X$. Then $X^{(n)}$ is a Dold space by induction on $n$ using Corollary 3.6. Hence $X$ is a Dold space by Proposition 3.8 (2).

Proposition 3.10 Let $p: E \rightarrow B$ be any map. Assume that $B$ and the homotopy fibers $F(p, b)$ of $p$ over $b$ are Dold spaces for all $b \in B$, then $E$ is a Dold space.

Proof By Proposition 3.1 we may assume $B$ is path-connected, and by Corollary 3.3 we may assume $p: E \rightarrow B$ is a fibration whose fiber $F$ over a fixed $b_{0} \in B$ is a Dold space. Let $\mathcal{U}=\left\{U_{\lambda} ; \lambda \in \Lambda\right\}$ be an open Dold cover of $B$ and $\left\{f_{\lambda}: B \rightarrow[0,1] ; \lambda \in \Lambda\right\}$ a numeration of $\mathcal{U}$, and let $\mathcal{V}=\left\{V_{\gamma}, \gamma \in \Gamma\right\}$ with $\left\{g_{\gamma}: F \rightarrow[0,1] ; \gamma \in \Gamma\right\}$ be the corresponding data for $F$. Let

$$
H_{\lambda}: U_{\lambda} \times I \rightarrow B
$$

be a homotopy from the inclusion $i_{\lambda}: U_{\lambda} \subset B$ to the constant map to $b_{0}$. Since $p$ is a fibration there is a homotopy $K_{\lambda}$

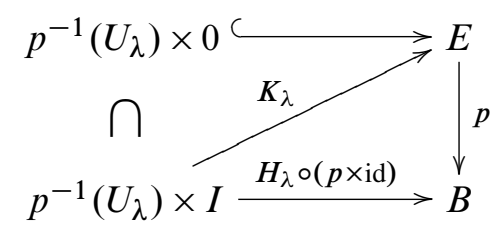

from the inclusion $j_{\lambda}: p^{-1}\left(U_{\lambda}\right) \subset E$ to a map $p^{-1}\left(U_{\lambda}\right) \stackrel{k_{\lambda}}{\rightarrow} F \subset E$. Define maps $\tau_{\lambda, \gamma}: E \rightarrow[0,1]$ by

$$
\tau_{\lambda, \gamma}(e)= \begin{cases}f_{\lambda}(p(e)) \cdot g_{\gamma}\left(k_{\lambda}(e)\right) & e \in p^{-1}\left(\operatorname{Supp}\left(f_{\lambda}\right)\right), \\ 0 & \left.e \notin p^{-1}\left(f_{\lambda}^{-1}(] 0,1\right]\right) .\end{cases}
$$

Since the $\left.\left.k_{\lambda}^{-1}\left(g_{\gamma}^{-1}(] 0,1\right]\right)\right), \gamma \in \Gamma$, cover $p^{-1}\left(U_{\lambda}\right)$ and since the $\left.\left.p^{-1}\left(f_{\lambda}^{-1}(] 0,1\right]\right)\right)$ $\subset p^{-1}\left(U_{\lambda}\right), \lambda \in \Lambda$, cover $E$,

$$
\left.\left.\mathcal{W}=\left\{W_{\lambda, \gamma}=\tau_{\lambda, \gamma}^{-1}(] 0,1\right]\right) ; \lambda \in \Lambda, \gamma \in \Gamma\right\}
$$

covers $E$. This cover is locally finite and ambiently contractible: $K_{\lambda}$ deforms $W_{\lambda, \gamma}$ into $k_{\lambda}\left(W_{\lambda, \gamma}\right) \subset V_{\gamma} \subset F$, and $V_{\gamma}$ is ambiently contractible in $F$. Let $e \in E$. Then there is an open neighborhood $U$ of $p(e)$ such that $U \cap U_{\lambda}=\varnothing$ for all but finitely many $\lambda_{1}, \ldots, \lambda_{n}$. So $p^{-1}(U)$ only meets $p^{-1}\left(U_{\lambda_{i}}\right), i=1, \ldots, n$. Each $k_{\lambda_{i}}(e)$ has 
an open neighborhood $V_{i}$ such that $V_{i} \cap V_{\gamma}=\varnothing$ for all but finitely many $\gamma_{i 1}, \ldots, \gamma_{i r_{i}}$. Then

$$
\left.\left.p^{-1}(U) \cap \bigcap_{i=1}^{n} k_{\lambda_{i}}^{-1}\left(V_{i}\right) \cap \tau_{\lambda, \gamma}^{-1}(] 0,1\right]\right) \neq \varnothing
$$

only if $(\lambda, \gamma) \in\left\{\left(\lambda_{i}, \gamma_{i j}\right) ; 1 \leq i \leq n, 1 \leq j \leq r_{i}\right\}$.

So $E$ is a Dold space by Corollary 2.9.

We now treat the based case.

Definition 3.11 We call a based space $\left(X, x_{0}\right)$ wellpointed, if the inclusion $\left\{x_{0}\right\} \subset X$ is a closed cofibration, and h-wellpointed if it is an h-cofibration.

The condition that $X$ be wellpointed can be achieved functorially by a whiskering process.

The whiskering process 3.12 For a based space $\left(X, x_{0}\right)$ we define $X_{I}=(X \sqcup I) /$ $\left(x_{0} \sim 1\right)$ and choose $0 \in I$ as basepoint of $X_{I}$. Then $X_{I}$ is wellpointed, and the natural map $q_{X}: X_{I} \rightarrow X$ mapping $I$ to $x_{0}$ is a homotopy equivalence. If $X$ is h-wellpointed, $q_{X}$ is even a based homotopy equivalence.

Proposition 3.13 Given a diagram

$$
X \stackrel{f}{\longleftarrow} A \stackrel{g}{\longrightarrow} Y
$$

of h-wellpointed spaces and based maps with $X$ and $Y$ Dold spaces. Then the reduced double mapping cylinder $M(f, g)$ is a Dold space.

Proof By the whiskering process we may assume that $A, X$, and $Y$ are wellpointed, because the reduced double cylinder construction is compatible with based homotopies. For wellpointed spaces the inclusion

$$
I \rightarrow \widehat{M}(f, g), \quad t \mapsto\left(a_{0}, t\right)
$$

of $I$ into the unreduced mapping cylinder is a cofibration. Hence the projection $\widehat{M}(f, g) \rightarrow M(f, g)$ is a homotopy equivalence.

Corollary 3.14 (1) The reduced suspension of an h-wellpointed space is a Dold space.

(2) The reduced join of $h$-wellpointed spaces is a Dold space. 
(3) Let $\left(\left(X_{\alpha}, x_{0}^{\alpha}\right) ; \alpha \in A\right)$ be a family of h-wellpointed Dold spaces. Then $\bigvee_{\alpha \in A} X_{\alpha}$ is a Dold space.

(4) If $X$ and $Y$ are $h$-wellpointed Dold spaces, then $X \wedge Y$ is a Dold space.

Proof (1) follows from Proposition 3.13, (2) holds because a reduced join is homotopy equivalent to a reduced suspension. For (3) note that $\bigsqcup_{\alpha} x_{0}^{\alpha} \subset \bigsqcup_{\alpha} X_{\alpha}$ is an $h-$ cofibration. Hence $\bigvee_{\alpha} X_{\alpha} \cong \bigsqcup_{\alpha} X_{\alpha} / \bigsqcup_{\alpha} x_{0}^{\alpha}$ is a Dold space by Corollary 3.7. For (4) we have the cofibration $X_{I} \vee Y_{I} \rightarrow X_{I} \times Y_{I}$. Hence $X_{I} \wedge Y_{I}$ is a Dold space by Corollary 3.7. Now use that $X \wedge Y \simeq X_{I} \wedge Y_{I}$.

Remark 3.15 Example 2.11 shows that Corollary 3.14 (3) does not hold without some assumptions on the basepoints.

We finally prove Proposition 1.6 (1) (see tom Dieck, Kamps and Puppe [4, (12.7)]):

Proposition 3.16 Let $X$ have a homotopy associative multiplication $\mu: X \times X \rightarrow X$ with homotopy unit $e$ such that $\mu$ induces a group structure on $\pi_{0}(X)$. Then

(1) the left and right translations $l_{x}, r_{x}: X \rightarrow X$ by $x \in X$ are homotopy equivalences.

(2) if $X$ is a Dold space, the shearing maps

$$
\begin{aligned}
& \operatorname{sh}_{1}: X \times X \rightarrow X \times X,(x, y) \mapsto(x, x \cdot y) \\
& \operatorname{sh}_{2}: X \times X \rightarrow X \times X,(x, y) \mapsto(x \cdot y, y)
\end{aligned}
$$

are homotopy equivalences.

(3) if $X$ is a Dold space, $\mu$ has a homotopy inverse inv: $X \rightarrow X$.

Proof (1) Since $\pi_{0}(X)$ is a group there is an $\bar{x} \in X$ such that $\bar{x} \cdot x$ is in the path-component of $e$. Since $\mu$ is homotopy associative we get

$$
l_{\bar{x}} \circ l_{x} \simeq l_{\bar{x} \cdot x} \simeq l_{e} \simeq \text { id and similarly } l_{x} \circ l_{\bar{x}} \simeq \mathrm{id} .
$$

(2) Consider the commutative diagram

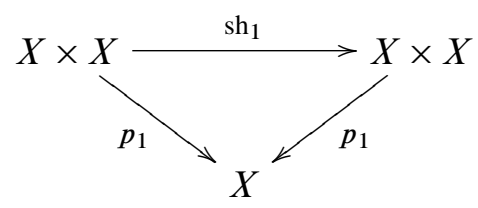

where $p_{i}$ is the projection onto the $i$-th factor. The restriction of $\operatorname{sh}_{1}$ to the fiber $p_{1}^{-1}(x)$ is left translation by $x$. Since $X$ is a Dold space, $p_{1}$ is a fibration, and each $l_{x}$ 
is a homotopy equivalence, the map $\mathrm{sh}_{1}$ is a fiberwise homotopy equivalence by Dold's Theorem 1.1.

(3) By (2) there is a fiberwise homotopy inverse $\varphi$ of $\operatorname{sh}_{1}$, and $x \mapsto p_{2} \circ \varphi(x, e)$ is a right homotopy inverse map.

\section{Homotopy pullbacks and the James construction}

The mapping path space of a map $g: B \rightarrow X$ is the subspace

$$
P(g)=\left\{(\omega, b) \in X^{I} \times B ; \omega(1)=g(b)\right\}
$$

of $X^{I} \times B$, where $X^{I}$ is the path space of $X$. The map

$$
P(g) \rightarrow X, \quad(\omega, b) \mapsto \omega(0)
$$

is a fibration. The double mapping path space of two maps $f: A \rightarrow X$ and $g: B \rightarrow X$

$$
P(f, g)=\left\{(a, \omega, b) \in A \times X^{I} \times B ; \omega(0)=f(a), \omega(1)=g(b)\right\}
$$

is then the pullback

$$
P(f, g)=A \times_{X} P(g) \cong P(f) \times_{X} B .
$$

Definition 4.1 A homotopy commutative diagram

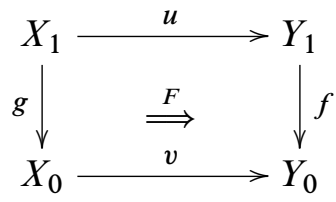

with commuting homotopy $F$ is called a homotopy pullback if the map

$$
r=\left(g, F^{\mathrm{ad}}, u\right): X_{1} \longrightarrow P(v, f) \subset X_{0} \times Y_{0}^{I} \times Y_{1},
$$

where $F^{\text {ad }}: X_{1} \rightarrow Y_{0}^{I}$ is the adjoint of $F: X_{1} \times I \rightarrow Y_{0}$, is a homotopy equivalence.

It is called a homotopy pushout if the map

$$
(v, F, f): \widehat{M}(g, v) \rightarrow Y_{0}
$$

is a homotopy equivalence. 
To simplify the exposition we restrict ourselves to strictly commuting squares, although the results also hold for homotopy commutative squares with specified commuting homotopies. The induced map $X_{1} \longrightarrow P(v, f)$ will usually be denoted by $r$. It factors as

$$
X_{1} \stackrel{\alpha}{\longrightarrow} P(u) \stackrel{r_{1}}{\longrightarrow} P(v, g),
$$

where $\alpha$ is the homotopy equivalence of the standard factorization of $u$ into a homotopy equivalence followed by a fibration, and the square above is a homotopy pullback if and only if $r_{1}$ is a homotopy equivalence.

We start with a result which is a consequence of Proposition 3.10.

Proposition 4.2 Given a commutative diagram

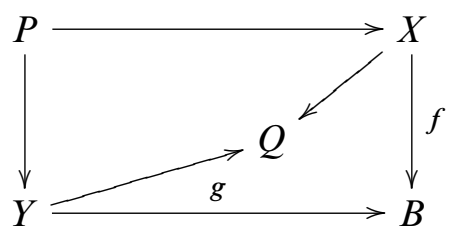

with $B$ a path-connected Dold space, whose outer square is a homotopy pullback and whose inner square is a homotopy pushout, then $Q$ is a Dold space.

Proof If $F(f)$ and $F(g)$ are the homotopy fibers of $f$ and $g$ respectively, the homotopy fiber of the induced map $r: Q \rightarrow B$ is homotopy equivalent to the join $F(f) *$ $F(g)$ (eg see Vogt [19, Proposition 5.5]). The result now follows from Proposition 3.10 .

Proposition 4.3 Let

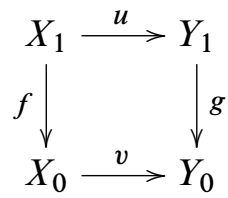

be a commutative square.

(1) If $v$ is a homotopy equivalence, then the square is a homotopy pullback if and only if $u$ is a homotopy equivalence.

(2) If the square is a homotopy pullback, $u$ is a homotopy equivalence, $g$ induces a surjection of sets of path-components, and $Y_{0}$ is a Dold space, then $v$ is a homotopy equivalence. 
Proof Consider the commutative diagram

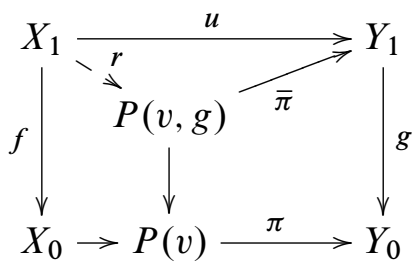

where $X_{0} \rightarrow P(v) \rightarrow Y_{0}$ is the standard factorization of $v$ into a homotopy equivalence followed by a fibration.

(1) Since $v$ is a homotopy equivalence, so is $\pi$. Since the inner square is a pullback with $\pi$ a fibration, $\bar{\pi}$ is a homotopy equivalence. Hence $u$ is a homotopy equivalence if and only if $r$ is a homotopy equivalence.

(2) Since $r$ and $u$ are homotopy equivalences $\bar{\pi}$ is a homotopy equivalence. Hence each fiber $F_{y}=\bar{\pi}^{-1}(y), y \in Y_{1}$ is contractible. Apply Dold's Theorem 1.1 to the diagram of fibrations

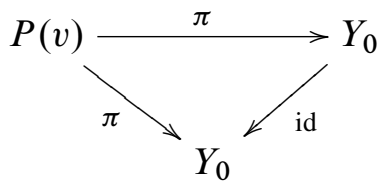

and use the fact that $\pi^{-1}(g(y)) \cong F_{y}$.

Example 4.4 Proposition 4.3 (2) does not hold if we drop the assumptions on $g$ and $Y_{0}$ : let

$$
\Omega C \stackrel{i}{\rightarrow} P C \longrightarrow C
$$

be the classical path-space fibration over the Warsaw circle $C$ with contractible $P C$. Then

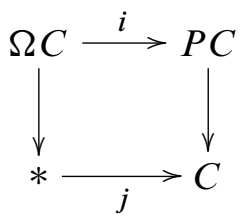

is a homotopy pullback, $i$ is a homotopy equivalence, but $j$ is not one.

\section{Proposition 4.5 Let}

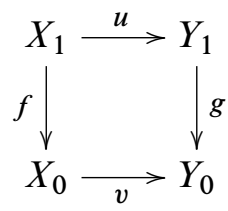

be a commutative square and $F(u, y)$ the homotopy fiber of $u$ over $y \in Y_{1}$. 
(1) If the square is a homotopy pullback, then the induced map

$$
F(u, y) \rightarrow F(v, g(y))
$$

is a homotopy equivalence for each $y \in Y_{1}$.

(2) If for each $y \in Y_{1}$ the map $F(u, y) \rightarrow F(v, g(y))$ is a homotopy equivalence and $Y_{1}$ is a Dold space, the square is a homotopy pullback.

Proof (1) Consider the commutative diagram:

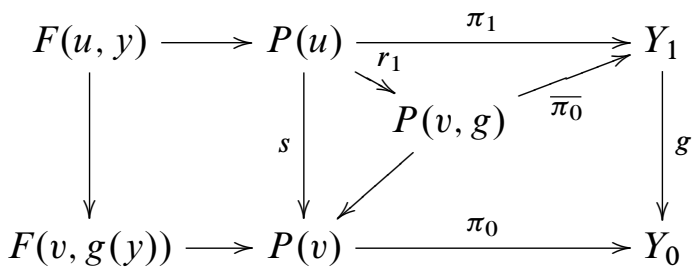

Since $\overline{\pi_{0}}$ and $\pi_{1}$ are fibrations, $r_{1}$ is a homotopy equivalence over $Y_{1}$, inducing a homotopy equivalence

$$
\begin{aligned}
F(u, y)=* \times_{Y_{1}} P(u) \rightarrow * \times_{Y_{1}} P(v, g) & =* \times_{Y_{1}} Y_{1} \times_{Y_{0}} P(v) \\
& \cong * \times_{Y_{0}} P(v)=F(v, g(y)) .
\end{aligned}
$$

(2) The map $F(u, y) \rightarrow F(v, g(y))$ factors as

$$
F(u, y)=\pi_{1}^{-1}(y) \rightarrow \bar{\pi}_{0}^{-1}(y) \rightarrow \pi_{0}^{-1}(g(y))=F(v, g(y)) .
$$

The second map is a homeomorphism. Hence $r_{1}$ is a fiberwise homotopy equivalence over $Y_{1}$ by Dold's theorem.

\section{Corollary 4.6 Let}

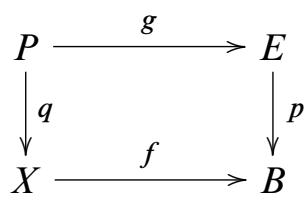

be a homotopy pullback, let $X$ and the homotopy fibers $F(p, b)$ of $p$ over all $b \in B$ be Dold spaces. Then $P$ is a Dold space.

Proof The homotopy fiber $F(q, x)$ of $q$ over $x \in X$ is homotopy equivalent to $F(p, f(x))$ and hence a Dold space. So $P$ is a Dold space by Proposition 3.10. 
Proposition 4.7 Consider the commutative diagram:

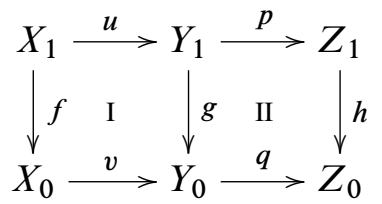

(1) Suppose that II is a homotopy pullback. Then I is a homotopy pullback if and only if the combined square I + II is a homotopy pullback

(2) Suppose that I and I + II are homotopy pullbacks, that $v$ induces a surjection of sets of path-components and $Y_{0}$ is a Dold space, then II is a homotopy pullback.

Proof Consider the commutative diagrams:

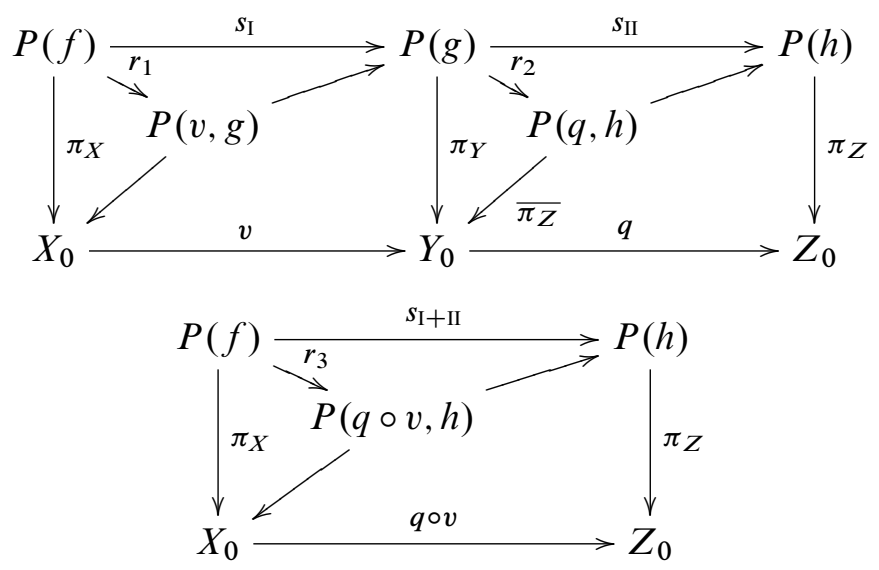

We obtain an induced commutative diagram

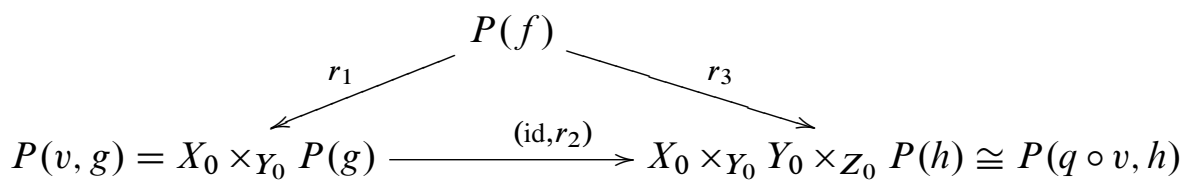

(1) We have to show that $r_{1}$ is a homotopy equivalence if and only if $r_{3}$ is one. By assumption $r_{2}$ is a homotopy equivalence over $Y_{0}$. Hence the horizontal map (id, $r_{2}$ ) of the previous diagram is a homotopy equivalence. 
(2) For each $x \in X_{0}$ we have a commutative diagram

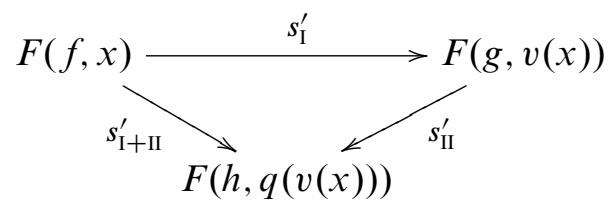

where the maps $s^{\prime}$ are the restrictions of the maps $s$ to the fibers. By Proposition 4.5 the maps $s_{\mathrm{I}}^{\prime}$ and $s_{\mathrm{I}+\mathrm{II}}^{\prime}$ are homotopy equivalences. Hence $s_{\mathrm{II}}^{\prime}$ is a homotopy equivalence for each $v(x) \in Y_{0}$. Since $v$ is surjective on path-components $s_{\text {II }}^{\prime}$ is a homotopy equivalence for each $y \in Y_{0}$. Now apply Proposition 4.5.

We will also make use of the first one of the two cube theorems:

\section{The cube theorems 4.8 Let}

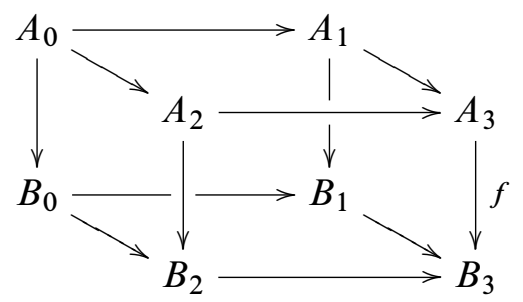

be a commutative cube.

The first cube theorem If the back and the left faces are homotopy pullbacks and the top and the bottom faces are homotopy pushouts then the right and the front faces are homotopy pullbacks.

The second cube theorem Suppose all vertical faces are homotopy pullbacks. Then

(1) the top face is a homotopy pushout if the bottom face is a homotopy pushout.

(2) the bottom face is a homotopy pushout if the top face is a homotopy pushout, $f$ induces a surjection on path-components, and $B_{3}$ is a Dold space.

Proof For a proof of the first cube theorem, refer to Puppe [15, Lemma 2] or Mather [9, Theorem 18]. For the first part of the second cube theorem, refer to Mather [9, Theorem 25].

We now prove Allaud's result using homotopy pullbacks:

Proposition 1.5 Let $f: X \rightarrow Y$ be a based map of path-connected Dold spaces such that $\Omega f: \Omega X \rightarrow \Omega Y$ is a homotopy equivalence. Then $f$ is a homotopy equivalence. 
Proof Consider the diagram

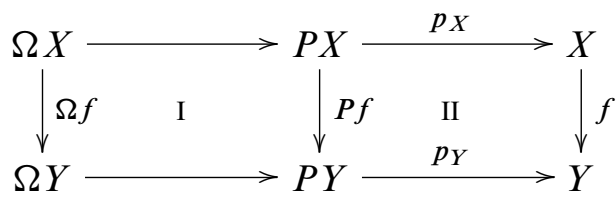

where the rows are the usual path-space fibrations. Since $\Omega f$ is a homotopy equivalence and $X$ is a Dold space, square II is a homotopy pullback by Proposition 4.5. Since $P X$ and $P Y$ are contractible, $P f$ is a homotopy equivalence. Since $Y$ is a path-connected Dold space $f$ is a homotopy equivalence by Proposition 4.3.

We next address an extension of Theorem 1.3.

For the rest of this section we work in $k$ Top* , the category of based $k$-spaces. Let TMON denote the category of topological monoids and continuous homomorphisms. The underlying space functor $U: T M O N \rightarrow k$ Top* has a left adjoint

$$
J: k \text { Top }^{*} \rightarrow T M O N,
$$

known as the James construction. For $(A, *) \in k$ Top $^{*}$ it is given by

$$
J A=\left(\coprod_{n \geq 1} A^{n}\right) / \sim
$$

with the relation

$$
\left(a_{1}, \ldots, a_{i}, \ldots, a_{n}\right) \sim\left(a_{1}, \ldots, a_{i-1}, a_{i+1}, \ldots, a_{n}\right) \quad \text { if } a_{i}=* .
$$

The multiplication is defined on representatives by

$$
\left(a_{1}, \ldots, a_{k}\right) \cdot\left(b_{1}, \ldots, b_{l}\right)=\left(a_{1}, \ldots, a_{k}, b_{1}, \ldots, b_{l}\right) .
$$

It is associative and has $(*)$ as neutral element.

A map $f: A \rightarrow B$ defines a map $J f: J A \rightarrow J B$ given on representatives by

$$
J f\left(a_{1}, \ldots, a_{n}\right)=\left(f\left(a_{1}\right), \ldots, f\left(a_{n}\right)\right)
$$

making $J$ into a functor. 
Let $f: A \rightarrow X$ be a based map and $M(f)$ the reduced mapping cylinder. Define $J(A, f)$ to be the pushout

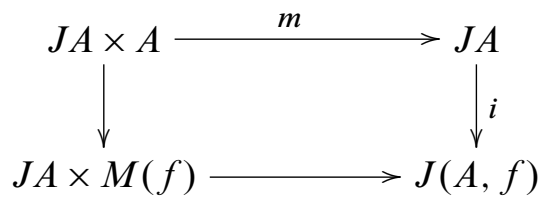

where $m: J A \times A \rightarrow J A$ is the multiplication.

Let $C(f)$ denote the reduced mapping cone of $f$. The projection

$$
J A \times M(f) \rightarrow M(f) \rightarrow C(f)
$$

induces a map

$$
q: J(A, f) \rightarrow C(f) .
$$

The following theorem is an extension of Theorem 1.3 and a reformulation D Puppe's theorem [14, Theorem 3.1].

Theorem 4.9 Let $f: A \rightarrow X$ be a based map of $h-$ wellpointed spaces.

(1) If $A$ is a path-connected Dold space, then the commutative diagram

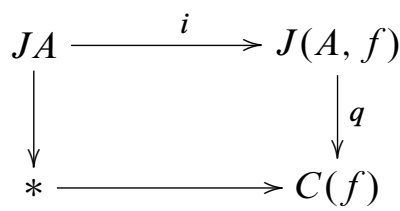

is a homotopy pullback.

(2) If $A$ and $X$ are path-connected Dold spaces, then there is a homotopy pullback

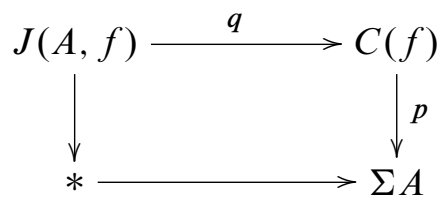

where $p: C(f) \rightarrow \Sigma A$ is the projection.

We will need:

Lemma 4.10 If $A$ is $h$-wellpointed and $X$ is a point then $J(A, f)$ is contractible. 
Proof The proof is due to D Puppe [14, Lemma 3.3]. We include it for the reader's convenience.

Due to the whiskering process 3.12 it suffices to prove the lemma for wellpointed spaces, because the James construction is compatible with based homotopies.

Note that $M(f)=C A$, the reduced cone on $A$. We filter $J(A, f)$ by word lengths, ie $J(A, f)_{n}$ is the image of $(J A)_{n} \times C A$ in $J(A, f)$. Then

$$
J(A, f)_{0}=C A \quad \text { and } \quad J(A, f)_{n} / J(A, f)_{n-1}=A \wedge \cdots \wedge A \wedge C A .
$$

Since $C A$ is contractible, all these spaces are contractible. Since $A$ is wellpointed $J(A, f)_{n-1} \subset J(A, f)_{n}$ is a closed cofibration. In particular,

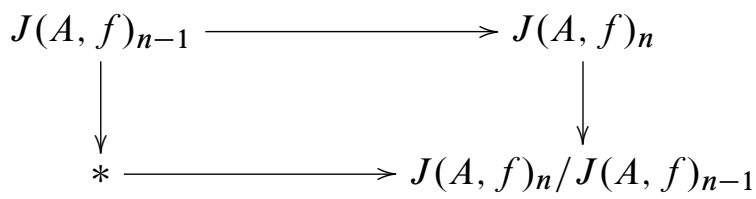

is a homotopy pushout. So, by induction and the dual of Proposition 4.3 (1),

$$
J(A, f)_{n} \rightarrow J(A, f)_{n} / J(A, f)_{n-1}
$$

is a homotopy equivalence. Hence each $J(A, f)_{n}$ is contractible. As colimit of a sequence of cofibrations between contractible spaces $J(A, f)$ is contractible.

Proof of Theorem 4.9 We may assume that $A$ and $X$ are wellpointed.

(1) The proof is essentially due to E Floyd [8, Theorem 6.19]. Consider the commutative diagram

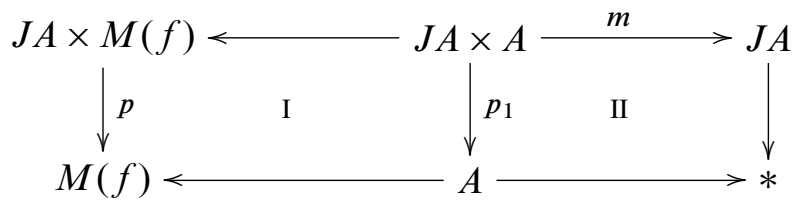

where $p$ and $p_{1}$ are projection maps. Square I is a pullback of fibrations and hence a homotopy pullback. Since $J A \rightarrow *$ is a fibration, square II is a homotopy pullback if and only if the induced map into the product

$$
\operatorname{sh}_{2}: J A \times A \rightarrow J A \times A, \quad(w, a) \mapsto(w \cdot a, a)
$$

is a homotopy equivalence. The restriction of $\operatorname{sh}_{2}$ to the fiber over $a \in A$ is right translation by $a$, which is homotopic to the identity, because $A$ is path-connected. By Dold's theorem $\mathrm{sh}_{2}$ is a homotopy equivalence. 
We now take pushouts of the rows to obtain a commutative cube. Since $J A \times A \rightarrow$ $J A \times M(f)$ and $A \rightarrow M(f)$ are cofibrations, the top and bottom faces of the cube are homotopy pushouts. Hence the result follows by the first cube theorem 4.8.

(2) The commutative square

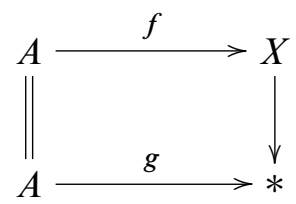

induces a map of homotopy pullbacks:

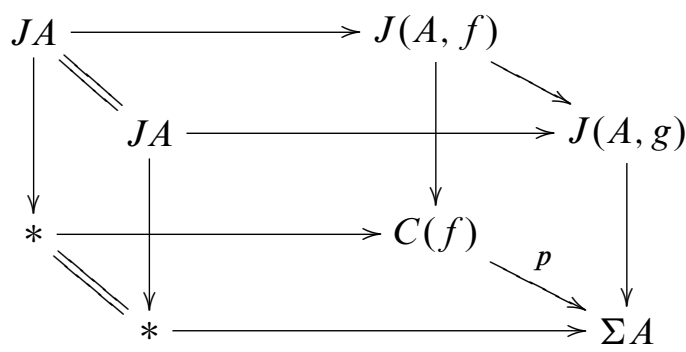

Since front and left side are homotopy pullbacks, the combined square

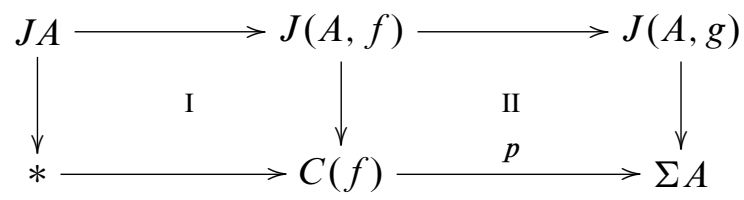

is a homotopy pullback. Since I is a homotopy pullback and $C(f)$ is a path-connected Dold space, II is a homotopy pullback by Proposition 4.7. By the lemma, $J(A, g) \simeq *$, hence the result follows.

We obtain Theorem 1.3 as a corollary.

Corollary 4.11 If $A$ is an $h$-wellpointed, path connected Dold space, there is a homotopy equivalence

$$
J A \rightarrow \Omega \Sigma A
$$

Proof Apply the theorem with $X=*$. Then $J(A, f) \simeq *$ and $C(f)=\Sigma A$. 


\section{Simplicial spaces}

Let $\triangle$ denote the category of finite ordered sets $[n]=\{0<1<\cdots<n\}$ and order preserving maps and Mon $\triangle$ the subcategory of injective order preserving maps. A simplicial space is a functor $X_{\bullet}: \triangle^{\mathrm{op}} \rightarrow$ Top, $[n] \mapsto X_{n}$, a semisimplicial space is a functor $X_{\bullet}:(\text { Mon } \triangle)^{\text {op }} \rightarrow$ Top.

Let $\left|X_{\bullet}\right|$ denote the usual topological realization, also called thin realization of the simplicial space $X_{\bullet}$, and $\left\|X_{\bullet}\right\|$ denote the realization of the semisimplicial space $X_{\bullet}$, also called fat realization. Since a simplicial space can be considered as a semisimplicial one, it has a fat and a thin realization. An element $x \in X_{n}$ is called degenerate if it is in the image of some $X_{\bullet}(\alpha)$ with $\alpha \neq$ id surjective. A simplicial space $X_{\bullet}$ is called proper if the inclusions $s X_{n} \subset X_{n}$ of the subspaces of degenerate elements are cofibrations for all $n$.

Proposition 5.1 (1) If $X_{\bullet}$ is a semisimplicial space such that $X_{0}$ is a Dold space, then $\left\|X_{\bullet}\right\|$ is a Dold space.

(2) If $X_{\bullet}$ is a proper simplicial space such that $X_{0}$ is a Dold space, then $\left|X_{\bullet}\right|$ is a Dold space.

Proof The following proof is due to D Puppe. We are indebted to $\mathrm{H}$ Meiwes for sending us the details [13].

(1) Let $\|X\|^{(n)}$ denote the $n$-skeleton of the fat realization. Since $\|X\|^{(n)} \subset\|X\|^{(n+1)}$ is a cofibration it suffices to show that each $\|X\|^{(n)}$ is a Dold space. Assume inductively that $\|X\|^{(n-1)}$ is a Dold space. Recall that

$$
\|X\|^{(n)}=\|X\|^{(n-1)} \cup_{X_{n} \times \partial \Delta^{n}} X_{n} \times \Delta^{n},
$$

where $\Delta^{n}$ is the standard $n$-simplex. Choose two different points $u_{1} \neq u_{2}$ in the interior of $\triangle^{n}$. For a space $Y$ let $C Y=(Y \times I) /(Y \times 0)$ be the cone on $Y$ with cone-point $*$, and $\varphi: C Y \rightarrow I$ the map $(y, t) \mapsto t$. Define maps

$$
\lambda_{i}:\left(\triangle^{n}, u_{i}\right) \stackrel{h_{i}}{\rightarrow}\left(C\left(\partial \triangle^{n}\right), *\right) \stackrel{\varphi}{\rightarrow}(I, 0) \quad i=1,2
$$

by choosing based homeomorphisms $h_{i}$ keeping the boundary fixed. The maps

$$
X_{n} \times \triangle^{n} \stackrel{\text { proj }}{\longrightarrow} \triangle^{n} \stackrel{\lambda_{i}}{\longrightarrow} I
$$

together with the constant map to 1 on $\|X\|^{(n-1)}$ define maps $f_{i}:\|X\|^{(n)} \rightarrow I$, and $\left.\left.\left.\left.\left\{f_{1}^{-1}(] 0,1\right]\right), f_{2}^{-1}(] 0,1\right]\right)\right\}$ is a numerable cover of $\|X\|^{(n)}$ by Lemma 2.8. The subspaces

$$
\left.\left.f_{i}^{-1}(] 0,1\right]\right)=\|X\|^{(n-1)} \cup_{X_{n} \times \partial \Delta^{n}} X_{n} \times\left(\triangle^{n} \backslash\left\{u_{i}\right\}\right)
$$


deformation retract onto $\|X\|^{(n-1)}$. Hence they are Dold spaces. So $\|X\|^{(n)}$ is a Dold space by Proposition 2.10 .

(2) If $X$ is a proper simplicial space the natural map $\|X\| \rightarrow|X|$ is a homotopy equivalence. Hence $|X|$ is a Dold space.

Corollary 5.2 The classifying space $B M$ of an $h$-wellpointed topological monoid $M$ is a Dold space.

Proof Recall that $B M=\left|N_{\bullet} M\right|$ where $N_{\bullet} M$ is the nerve of $M$. Since the classifying space construction is compatible with based homotopies, we may assume that $M$ is wellpointed by the whiskering process 3.12. (Extend the multiplication of $M$ to $M_{I}$ by $x \cdot t_{1}=t_{1} \cdot x=x$ and $\max \left\{t_{1}, t_{2}\right\}$ for $x \in M$ and $t_{1}, t_{2} \in I$.) But then $N_{\bullet} M$ is a proper simplicial space. The result follows from Proposition 5.1 because $N_{0} M$ is a point.

Proposition 5.3 Let $J$ be a small category and $D: J \rightarrow$ Top a diagram of Dold spaces. Then hocolim $D$ is a Dold space.

Proof hocolim $D$ is the topological realization of the proper simplicial space

$$
[n] \mapsto \coprod_{i, j \in J} J_{n}(i, j) \times D(i)
$$

with $J_{n}(i, j)=\left\{\left(\alpha_{1}, \ldots, \alpha_{n}\right) \in(\operatorname{mor} J)^{n} ; \alpha_{1} \circ \cdots \circ \alpha_{n}: i \rightarrow j\right\}$ for $n>0$ and

$$
J_{0}(i, j)=\left\{\begin{array}{cc}
\text { id } & i=j \\
\varnothing & i \neq j
\end{array}\right.
$$

Its 0 -th space is $\bigsqcup_{j \in J} D(j)$ and hence a Dold space.

We now consider the based case. The homotopy colimit of a diagram $D$ in the category of based spaces is the reduced homotopy colimit hocolim* $D$ which in general is not homotopy equivalent to hocolim $D$. Let $B J$ denote the classifying space of $J$. The inclusions of the basepoints define a map

$$
B J \rightarrow \operatorname{hocolim} D,
$$

and hocolim* $D=(\operatorname{hocolim} D) / B J$.

Proposition 5.4 Let $D: J \rightarrow$ Top* be a diagram of $h$-wellpointed Dold spaces and based maps. Then hocolim* $D$ is a Dold space.

Proof Since the reduced homotopy colimit is compatible with based homotopies, we may assume that $D$ is a diagram of wellpointed spaces by the whiskering process 3.12 . But then $B J \rightarrow$ hocolim $D$ is a cofibration. Now apply Corollary 3.7. 
Example 5.5 Recall that a closed class $\mathcal{C}$ in the sense of Dror Farjoun [6, 2.D.1] is a full subcategory of the category $S_{*}$ of wellpointed spaces of the homotopy type of a $\mathrm{CW}$-complex which is closed under homotopy equivalences and reduced homotopy colimits.

The class of wellpointed Dold spaces is closed under homotopy equivalences and reduced homotopy colimits, but Dror Farjoun's results do not generalize to this class: Let $F \rightarrow E \rightarrow B$ be a fibration sequence with path-connected $B$. If $F$ and $E$ are in a closed class $\mathcal{C}$, then so is $B$ [6, 2.D.11].

This does not hold for Dold spaces. Consider the usual path-space fibration

$$
\Omega C \rightarrow P C \rightarrow C
$$

over the Warsaw circle $C$ with a nice point $c_{0} \in C$. It is well-known that $\Omega C$ and $P C$ are contractible and hence Dold spaces, but $C$ is not a Dold space.

We next give a characterization of path-connected Dold spaces which needs some preparations. For a based space $\left(X, x_{0}\right)$ let $\operatorname{Sing}_{\bullet}^{0} X$ denote the simplicial space whose $n$-th space $\operatorname{Sing}_{n}^{0} X$ is the space of maps $\left(\Delta^{n}, \Delta_{0}^{n}\right) \rightarrow\left(X, x_{0}\right)$, where $\Delta_{0}^{n}$ is the $0-$ skeleton of the $n$-simplex $\Delta^{n}$. Boundaries and degeneracies are defined as for the singular functor.

The evaluation maps $\operatorname{Sing}_{n}^{0} X \times \Delta^{n} \rightarrow X,(\sigma, t) \mapsto \sigma(t)$ induce a map

$$
\text { ev: }\left\|\operatorname{Sing}_{\bullet}^{0} X\right\| \rightarrow X \text {. }
$$

Proposition 5.6 A path-connected based space $X$ is a Dold space if and only if ev: $\left\|\operatorname{Sing}_{\bullet}^{0} X\right\| \rightarrow X$ is a homotopy equivalence.

Remark 5.7 We cannot replace $\operatorname{Sing}_{\bullet}^{0} X$ by $\operatorname{Sing}_{\bullet} X$, the usual topologized singular functor, because $\left\|\operatorname{Sing}_{\bullet} X\right\| \simeq X$ for any $X$ : the maps $[n] \rightarrow[0]$ in $\Delta$ induce a simplicial map $\pi_{\bullet}: c_{\bullet} X \rightarrow$ Sing $X$ from the constant simplicial space on $X$ to Sing $X$. Since each $\pi_{n}$ is a homotopy equivalence $\left\|\pi_{\bullet}\right\|:\left\|c_{\bullet} X\right\| \rightarrow \|$ Sing $X \|$ is a homotopy equivalence, and $X \simeq\left\|c_{\bullet} X\right\|$.

We postpone the proof of Proposition 5.6 a little.

Let

$$
p: E \rightarrow X
$$

be a map of based spaces with path-connected $X$ and $F(p)$ the homotopy fiber of $p$ over the basepoint $x_{0}$. With $p$ we associate a map

$$
q_{\bullet}: E_{\bullet}(p) \rightarrow \operatorname{Sing}_{\bullet}^{0} X
$$


of simplicial spaces as follows: Let $C \triangle^{n}$ denote the cone on $\triangle^{n}$ with cone point $c_{0}$. We define

$$
E_{n}(p)=\left\{(e, w) \in E \times \operatorname{Top}\left(\left(C \triangle^{n}, \triangle_{0}^{n}\right),\left(X, x_{0}\right)\right) ; w\left(c_{0}\right)=p(e)\right\} .
$$

Boundaries and degeneracies are defined by the corresponding maps of the standard simplices just like for the singular functor. We define

$$
q_{n}: E_{n}(p) \rightarrow \operatorname{Sing}_{n}^{0} X, \quad(e, w) \mapsto w \mid \triangle^{n} .
$$

Let $L_{n} \subset \triangle^{n}$ be the union of edges joining the $i$-th with the $(i+1)$-st vertex of $\triangle^{n}$. Since $L_{n} \subset \triangle^{n}$ is a strong deformation retract and the inclusion is a cofibration there is a fibration and homotopy equivalence

$$
\operatorname{Sing}_{n}^{0} X \rightarrow \operatorname{Top}\left(\left(L_{n}, L_{n} \cap \triangle_{0}^{n}\right),(X, *)\right) \cong(\Omega X)^{n} .
$$

Since $C \triangle^{n} \cong \triangle^{n+1}$ we have a similar homotopy equivalence

$$
E_{n}(p) \rightarrow F(p) \times(\Omega X)^{n},
$$

and

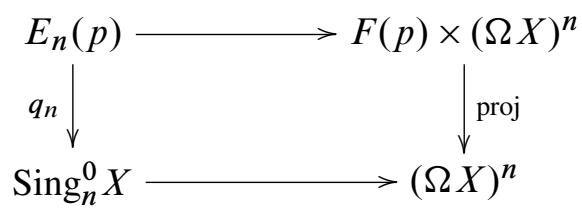

commutes. Using this it is easy to show that $q_{\bullet}: E_{\bullet}(p) \rightarrow \operatorname{Sing}_{\bullet}^{0} X$ is a simplicial object in the category Pull, whose objects are maps and whose maps are commutative squares which are homotopy pullbacks. A result of V Puppe [15, Theorem] implies

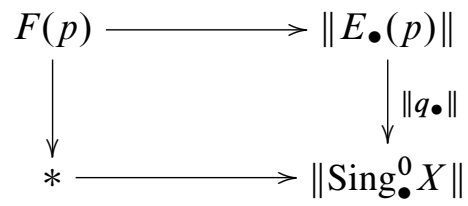

is a homotopy pullback. The horizontal maps are the inclusions of the 0 -skeleta.

Let $P(p)=\left\{(e, \alpha) \in E \times X^{I} ; \alpha(0)=p(e)\right\}$ be the mapping path-space of $p$. The maps

$$
E_{n}(p) \times \Delta^{n} \rightarrow P(p), \quad(e, w, t) \mapsto(e, \bar{w})
$$

with $\bar{w}(s)=w(s, t)$ for $(s, t) \in C \triangle^{n}=\left(I \times \triangle^{n}\right) /\left(0 \times \triangle^{n}\right)$, define a map

$$
u:\left\|E_{\bullet}(p)\right\| \longrightarrow P(p) .
$$


We obtain a map of fiber sequences

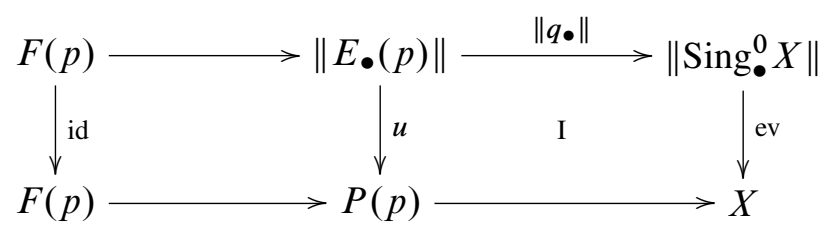

Since $\left\|\operatorname{Sing}_{\bullet}^{0} X\right\|$ is a Dold space by Proposition 5.1, square I is a homotopy pullback by Proposition 4.5.

Proof of Proposition 5.6 Since $\left\|\operatorname{Sing}_{\bullet}^{0} X\right\|$ is a Dold space so is $X$ if ev is a homotopy equivalence. For the converse take $E$ to be a point. Then $P(p)$ is contractible, and so is $\left\|E_{\bullet}(p)\right\|$ : Note that $E_{n}(p) \cong \operatorname{Sing}_{n+1}^{0} X$, so that $E_{\bullet}(p)$ is the simplicial based path-space construction $P\left(\operatorname{Sing}_{\bullet}^{0} X\right)$ in Top ${ }^{{ }^{\text {op }}}$ whose fat realization $\left\|P\left(\operatorname{Sing}_{\bullet}^{0} X\right)\right\|$ is known to be homotopy equivalent to $\operatorname{Sing}_{0}^{0} X=*$ (eg see Segal [16, page 296]). So $u$ is a homotopy equivalence. Hence ev is a homotopy equivalence by Proposition 4.3, if $X$ is a path-connected Dold space.

Corollary 5.9 If $X$ is a path-connected Dold space, then for any based map $p: E \rightarrow X$ the maps $u$ and ev of (5.8) are homotopy equivalences.

Remark 5.10 From Corollary 5.9 we obtain an alternative proof of Proposition 3.10. Let $p: E \rightarrow X$ be a map, $X$ a path-connected Dold space, and suppose the homotopy fiber $F(p)$ is also a Dold space, then $E$ is a Dold space: Consider

$$
\left\|E_{\bullet}(p)\right\| \stackrel{u}{\rightarrow} P(p) \stackrel{r}{\rightarrow} E
$$

with $r(e, \alpha)=e$. The maps $u$ and $r$ are homotopy equivalences. Since $E_{0}(p)=F(p)$, the space $\left\|E_{\bullet}(p)\right\|$ is a Dold space by Proposition 5.1, and hence so is $E$.

We will now apply our set-up to simplicial maps.

Proposition 5.11 Let $p_{\bullet}: E_{\bullet} \rightarrow X_{\bullet}$ be a map of based semisimplicial spaces. Let $F\left(p_{n}\right)$ denote the homotopy fiber of $p_{n}: E_{n} \rightarrow X_{n}$. If each $X_{n}$ is a path-connected Dold space, then

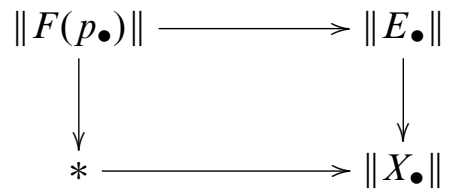

is a homotopy pullback. 
Proof We apply our construction to each map $p_{n}: E_{n} \rightarrow X_{n}$. This gives us bisemisimplicial spaces, ie functors

$$
X_{\bullet \diamond:}(\text { Mon } \triangle)^{\mathrm{op}} \times(\text { Mon } \triangle)^{\mathrm{op}} \rightarrow \text { Top, } \quad([k],[n]) \mapsto X_{k n} .
$$

Since our construction is natural we have a commutative diagram of semisimplicial spaces

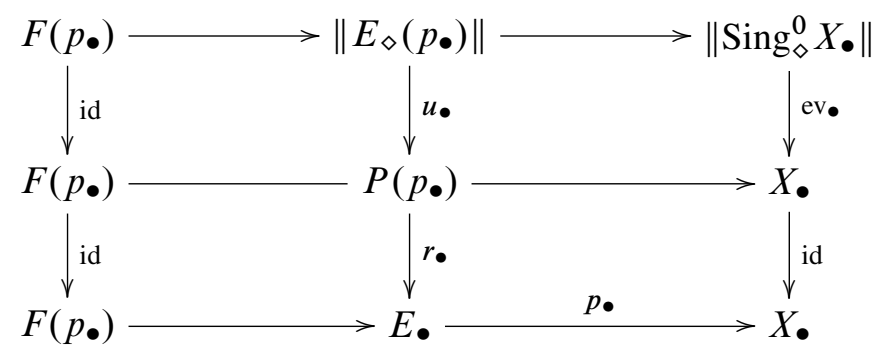

where the realizations are taken with respect to $\diamond$. The vertical maps are homotopy equivalences in each degree and hence induce homotopy equivalences of fat realizations. So it suffices to show that

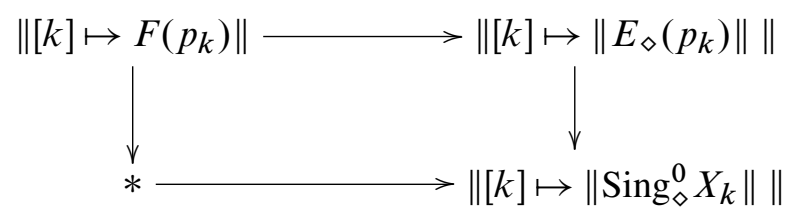

is a homotopy pullback. For this we study the map

$$
q_{n, k}: E_{n}\left(p_{k}\right) \rightarrow \operatorname{Sing}_{n}^{0}\left(p_{k}\right)
$$

of bisemisimplicial spaces. Since its total fat realization is independent of the order in which we realize, we may first realize with respect to $k$ and obtain a map of semisimplicial spaces

$$
\bar{q}_{n}:\left\|E_{n}\left(p_{\bullet}\right)\right\| \rightarrow\left\|\operatorname{Sing}_{n}^{0} X_{\bullet}\right\|
$$

Claim $\bar{q}_{n}$ is a semisimplicial object in Pull, ie

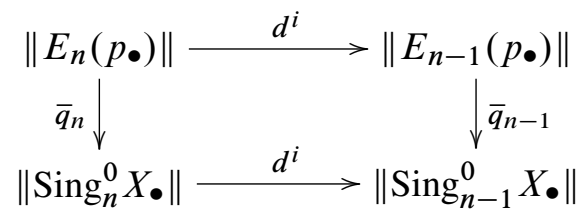

is a homotopy pullback for each $n$ and $i$.

Proof Let $j \neq i$. There is a strong deformation retraction of $C \triangle^{n}$ to $\triangle^{n} \cup \bigcup_{v_{j}}\left[c_{0}, v_{j}\right]$, where $\left[c_{0}, v_{j}\right]$ is the line from the cone point $c_{0}$ to the $j$-th vertex $v_{j}$ of $\triangle^{n}$. This 
deformation retraction can be chosen compatibly with $d^{i}$ yielding a commutative square

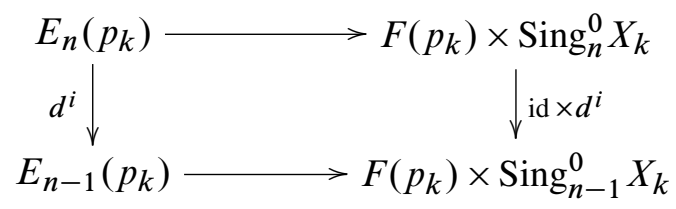

whose horizontal maps are homotopy equivalences. Since the fat realization preserves products up to homotopy, we obtain a commutative diagram

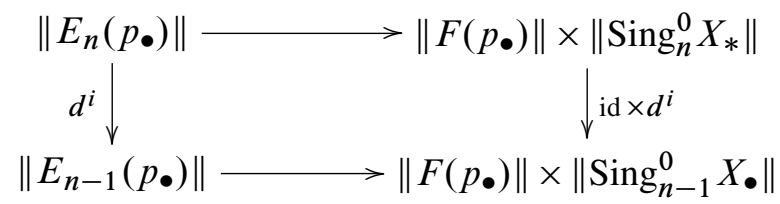

whose horizontal maps are homotopy equivalences. So it suffices to show that

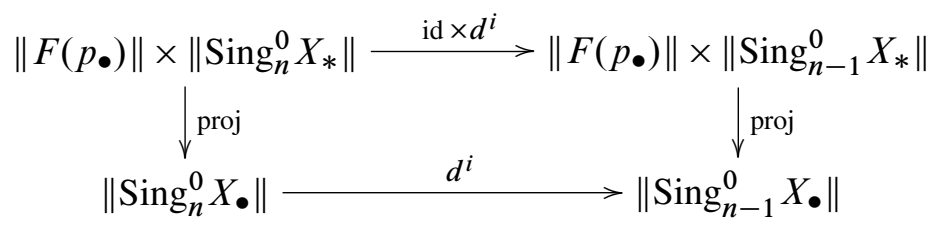

is a homotopy pullback. But this is evident. This proves the claim.

We now apply V Puppe's result [15] again: Since $\left\|F\left(p_{\bullet}\right)\right\|$ is the 0 -skeleton of $\left\|[n] \mapsto E_{n}\left(p_{\bullet}\right)\right\|$, we obtain a homotopy pullback:

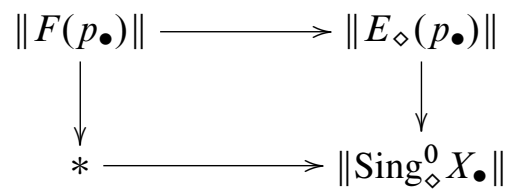

This completes the proof of Proposition 5.11.

As an immediate consequence we obtain:

Proposition 5.12 Let $X_{\bullet}$ be a based semisimplicial space such that each $X_{n}$ is a path-connected Dold space. Then there is a canonical homotopy equivalence

$$
\left\|\Omega X_{\bullet}\right\| \rightarrow \Omega\left\|X_{\bullet}\right\| .
$$

In particular, $\Omega\left\|X_{\bullet}\right\|$ is a Dold space if $\Omega X_{0}$ is a Dold space (eg if $X_{0}$ is based contractible). Here $\Omega X_{\bullet}$ is the semisimplicial space $[n] \mapsto \Omega X_{n}$. 
Proof Let $*$ denote the semisimplicial point. Apply Proposition 5.11 to the map $p_{\bullet}: * \rightarrow X_{\bullet}$. Since $F\left(p_{\bullet}\right)=\Omega X_{\bullet}$ and $\|*\|$ is contractible, the statement follows.

Remark 5.13 The proofs of the previous two propositions are based on ideas of D Puppe who sketched a proof of Proposition 5.12 to the second author during a private conversation. Instead of Sing. ${ }^{0} X$ Puppe used the nerve $N_{\bullet} \Omega_{M} X$ of the Moore loop space $\Omega_{M} X$ of $X$. There is a simplicial map [18, Lemma 2.8]

$$
\alpha_{\bullet}: N_{\bullet} \Omega_{M} X \rightarrow \operatorname{Sing}_{\bullet}^{0} X
$$

which is degreewise a homotopy equivalence inducing a homotopy equivalence

$$
\left\|\alpha_{\bullet}\right\|:\left\|N_{\bullet} \Omega_{M} X\right\| \rightarrow\left\|\operatorname{Sing}_{\bullet}^{0} X\right\| .
$$

We note that $N_{\bullet} \Omega_{M} X$ is proper if $X$ is wellpointed, because $\Omega_{M} X$ is wellpointed respectively h-wellpointed if $X$ is [4, (11.3)].

The remark and the whiskering process 3.12 imply:

Proposition 5.14 If $X$ is an $h$-wellpointed path-connected space, then $X$ is a Dold space if and only if ev o $\left|\alpha_{*}\right|: B \Omega_{M} X \rightarrow X$ is a based homotopy equivalence, where $B$ is the classifying space functor.

In general, loop spaces of Dold spaces need not be Dold spaces as the following example shows:

Example 5.15 $\mathbb{Q}$ with the subspace topology of $\mathbb{R}$ is not a Dold space. Let $N \cdot \mathbb{Q}$ denote the nerve of $(\mathbb{Q},+)$. Since $(\mathbb{Q},+)$ is a topological group there is a homotopy equivalence $\mathbb{Q} \rightarrow \Omega\|N \bullet \mathbb{Q}\|$. Hence $\Omega\|N \bullet \mathbb{Q}\|$ is not a Dold space but $\left\|N_{\bullet} \mathbb{Q}\right\|$ is one.

We can show:

Proposition 5.16 If $X$ is a path-connected h-wellpointed Dold space, then $\Omega \Sigma X$ is a Dold space.

Proof By the whiskering process 3.12 we may assume that $X$ is wellpointed. We make use of the dual $W_{\bullet}(X)$ of the better known cobar construction. $W_{\bullet}(X)$ is the simplicial space where $W_{n}(X)$ is the $n$-fold wedge of $X$. The boundary maps $d^{i}: W_{n}(X) \rightarrow W_{n-1}(X)$ are given by deleting the first respectively the last wedge summand if $i=0$ respectively $i=n$. For $0<i<n$ they are given by applying the fold map to the $i$-th and $i+1-$ st wedge summand. The $i-$ th degeneracy map 
maps $\left(x_{1}, \ldots, x_{n}\right)$ to $\left(x_{1}, \ldots, *, x_{i}, \ldots, x_{n}\right)$ where $*$ is the basepoint of $X$. Since all elements of $W_{n}(X)$ are degenerate for $n \geq 2$ we have $\left|W_{\bullet}(X)\right| \cong \Sigma X$.

Apply Proposition 5.11 to $p_{\bullet}: * \rightarrow W_{\bullet}(X)$. Since $W_{\bullet}(X)$ is proper, we have homotopy equivalences

$$
\left\|F\left(p_{\bullet}\right)\right\|=\left\|\Omega W_{\bullet}(X)\right\| \simeq \Omega\left\|W_{\bullet}(X)\right\| \simeq \Omega\left|W_{\bullet}(X)\right| \cong \Omega \Sigma X .
$$

Since $F\left(p_{0}\right)$ is a point, $\left\|F\left(p_{\bullet}\right)\right\|$ and hence $\Omega \Sigma X$ is a Dold space.

We close this section with an extension of Proposition 5.11.

Proposition 5.17 Given a commutative diagram of based semisimplicial spaces

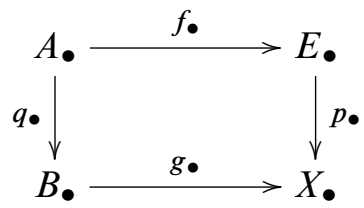

which is a homotopy pullback in each dimension. If each $B_{n}$ and each $X_{n}$ is a path-connected Dold space, then

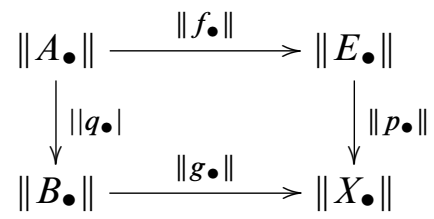

is a homotopy pullback.

Proof From Proposition 5.11 we obtain a diagram

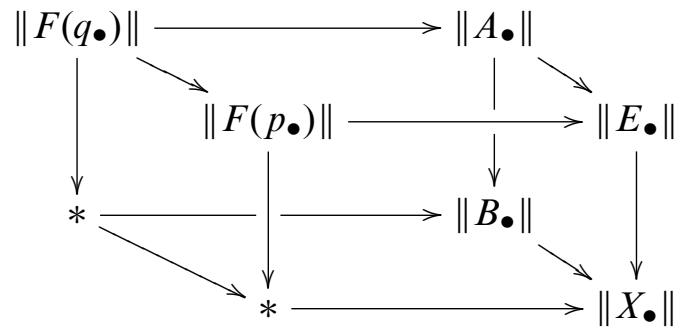

whose front face $(F)$ and back face $(B)$ are homotopy pullbacks. Since the map $F\left(q_{\bullet}\right) \rightarrow F\left(p_{\bullet}\right)$ is a homotopy equivalence in each dimension by assumption, its realization is a homotopy equivalence. Hence the left face $(L)$ is a homotopy pullback. If $(R)$ denotes the right face, we find that $(B)+(R)$ is a homotopy pullback, because $(L)+(F)$ is one. Hence $(R)$ is a homotopy pullback by Proposition 4.7. 


\section{6 k-spaces and free algebras over operads}

Throughout this section we work in the category $k$ Top of $k$-spaces and its based version $k$ Top* . Recall that $X$ is a $k$-space if a subset $U \subset X$ is open precisely if $f^{-1}(U)$ is open for all maps $f: C \rightarrow X$ and all compact Hausdorff spaces $C$. The inclusion functor $i: k$ Top $\subset$ Top has a right adjoint $k$ : Top $\rightarrow k$ Top obtained from $X$ by declaring the subsets $U$ satisfying the condition above as open. Hence the topology of $k(X)$ is finer than the one of $X$, and we obtain:

Proposition 6.1 If $X$ is a Dold space so is $k(X)$.

Since $i$ preserves colimits and $k$ limits, we moreover have:

Proposition 6.2 The results of the previous sections also hold in the category $k$ Top respectively $k$ Top*.

We include $k$ Top into our considerations because Theorems 1.3 and 1.4 are phrased in $k$ Top*.

In his proof of Theorem 1.4 Meiwes needed to show that $\mathcal{C}_{n}^{*}(X)$ and the $k$-fold symmetric product $\operatorname{SP}_{k}(X)=X^{k} / \Sigma_{k}$ of $X$ are Dold spaces if $X$ is a $(h$-wellpointed for $\left.\mathcal{C}_{n}^{*}(X)\right)$ Dold space. He did this by explicitly constructing Dold covers. We will obtain these results from more general easy to prove facts.

Let $\mathscr{P}$ be a topological operad. We call $\mathscr{P}$ reduced if $\mathscr{P}(0)$ consists of a single element. If $X$ is a $\mathscr{P}$-space and $\mathscr{P}$ is reduced, the single element of $\mathscr{P}(0)$ determines a basepoint in $X$. Let $\mathcal{P}$ Top be the category of $\mathscr{P}$-spaces. We have forgetful functors

$$
U: \mathcal{P} \text { Top } \rightarrow k \text { Top }
$$

and, if $\mathscr{P}$ is reduced,

$$
U^{*}: \mathcal{P} \text { Top } \rightarrow k \text { Top* }
$$

They have left adjoints

$$
\mathcal{P}: k \text { Top } \rightarrow \mathcal{P} \text { Top, } \quad \text { respectively, } \quad \mathcal{P}^{*}: k \text { Top }^{*} \rightarrow \mathcal{P} \text { Top }
$$

defined by

$$
\mathcal{P}(X)=\coprod_{n=0}^{\infty} \mathscr{P}(n) \times \Sigma_{\Sigma_{n}} X^{n} \quad \text { and } \quad \mathcal{P}^{*}(X)=\left(\coprod_{n=0}^{\infty} \mathscr{P}(n) \times_{\Sigma_{n}} X^{n}\right) / \sim
$$


The relation $\sim$ in the definition of $\mathcal{P}^{*}(X)$ is defined as follows: Let $* \in \mathscr{P}(0)$ denote the single element and $* \in X$ the basepoint, and let

$$
\begin{aligned}
\sigma_{i}: \mathscr{P}(k) \rightarrow \mathscr{P}(k-1), & \alpha \mapsto \alpha \circ\left(\mathrm{id}_{i} \times * \times \mathrm{id}_{k-i-1}\right) \\
s_{i}: X^{k-1} \rightarrow X^{k}, & \left(x_{1}, \ldots, x_{k-1}\right) \mapsto\left(x_{1}, \ldots, x_{i}, *, x_{i+1}, \ldots, x_{k-1}\right) .
\end{aligned}
$$

Then $\left(\sigma_{i}(\alpha), x\right) \sim\left(\alpha, s_{i}(x)\right)$.

Proposition 6.3 Let $\mathscr{P}$ be an operad such that each $\mathscr{P}(n) / \Sigma_{n}$ is a Dold space, and let $X \in k$ Top be a path-connected Dold space. Then $\mathcal{P}(X)$ is a Dold space.

Proposition 6.4 Let $X \in k$ Top* be an $h$-wellpointed path-connected Dold space. Then $\mathcal{P}^{*}(X)$ is a Dold space for each reduced operad $\mathscr{P}$.

The proofs make use of the following result of May [10, Theorem 12.2]:

Proposition 6.5 (1) Let $X_{\bullet}$ be a simplicial $k$-space, then there is a natural homeomorphism $\left|\mathcal{P}\left(X_{\bullet}\right)\right| \rightarrow \mathcal{P}\left(\left|X_{\bullet}\right|\right)$.

(2) Let $X_{\bullet}$ be a wellpointed simplicial $k$-space and $\mathscr{P}$ a reduced operad. Then there is a natural homeomorphism $\left|\mathcal{P}^{*}\left(X_{\bullet}\right)\right| \rightarrow \mathcal{P}^{*}\left(\left|X_{\bullet}\right|\right)$.

May proves the based case, but the proof applies verbatim also to the nonbased case.

Proof of Proposition 6.3 Choose a basepoint $x_{0} \in X$ and let $q_{X}: X_{I} \rightarrow X$ be the homotopy equivalence of the whiskering process. Since $X_{I}$ is a wellpointed Dold space the map

$$
\mathrm{ev} \circ\left|\alpha_{\bullet}\right|:\left|N_{\bullet} \Omega_{M} X_{I}\right| \rightarrow X_{I}
$$

of Proposition 5.14 is a based homotopy equivalence. By Proposition 6.5 we have a sequence of homotopy equivalences (we ignore basepoints)

$$
\left|\mathcal{P}\left(N_{\bullet} \Omega_{M} X_{I}\right)\right| \rightarrow \mathcal{P}\left(\left|N_{\bullet} \Omega_{M} X_{I}\right|\right) \rightarrow \mathcal{P}\left(X_{I}\right) \rightarrow \mathcal{P}(X) .
$$

$N_{0} \Omega_{M} X_{I}$ is a single point. Hence

$$
\mathcal{P}\left(N_{0} \Omega_{M} X_{I}\right)=\mathcal{P}(*)=\coprod_{n=0}^{\infty} \mathcal{P}(n) / \Sigma_{n}
$$

which is a Dold space. Hence $\left|\mathcal{P}\left(N_{\bullet} \Omega_{M} X_{I}\right)\right|$ and $\mathcal{P}(X)$ are Dold spaces by Proposition 5.1, because $\mathcal{P}\left(N_{\bullet} \Omega_{M} X_{I}\right)$ is proper. 
Proof of Proposition 6.4 If $X$ is $h$-wellpointed the map $q: X_{I} \rightarrow X$ is a based homotopy equivalence. Since the construction $\mathcal{P}^{*}$ is compatible with based homotopies we obtain a sequence of based homotopy equivalences

$$
\left|\mathcal{P}^{*}\left(N_{\bullet} \Omega_{M} X_{I}\right)\right| \rightarrow \mathcal{P}^{*}\left(\left|N_{\bullet} \Omega_{M} X_{I}\right|\right) \rightarrow \mathcal{P}^{*}\left(X_{I}\right) \rightarrow \mathcal{P}^{*}(X) .
$$

Since $\mathcal{P}^{*}(*)=*$, all spaces are Dold spaces by Proposition 5.1, as $\mathcal{P}^{*}\left(N_{\bullet} \Omega_{M} X_{I}\right)$ is proper.

Corollary 6.6 The $n$-fold symmetric product $\operatorname{SP}_{n}(X)$ of a Dold space $X \in k$ Top is a Dold space.

Proof Let $\left\{X_{\alpha} ; \alpha \in A\right\}$ be the set of path-components of $X$. Then $\operatorname{SP}_{n}(X)$ is the disjoint union of spaces

$$
\operatorname{SP}_{r_{1}}\left(X_{\alpha_{1}}\right) \times \cdots \times \operatorname{SP}_{r_{q}}\left(X_{\alpha_{q}}\right), \quad r_{1}+\cdots+r_{q}=n .
$$

By Proposition 3.1 and Examples 2.5 (6) it suffices to prove the result for pathconnected $X$.

Let $\mathscr{C o m}$ be the operad for commutative monoid structures, ie $6 \mathrm{om}(r)$ is a single point for each $r$. Then

$$
\operatorname{Com}(X)=\coprod_{n} \operatorname{SP}_{n}(X)
$$

is a Dold space by Proposition 6.3. $\mathrm{So}_{n}(X)$ is a Dold space by Proposition 3.1.

Remark 6.7 Proposition 6.4 provides an alternative proof of Proposition 5.16 in the category of $k$-spaces. Let Mon be the operad for monoid structures and $X$ an $h$-wellpointed path-connected $k$-space. Then $\mathcal{M o n}^{*}(X)=J X$ is a Dold space by Proposition 6.4, and $J X \simeq \Omega \Sigma X$ by Corollary 4.11.

\section{References}

[1] G Allaud, De-looping homotopy equivalences, Arch. Math. (Basel) 23 (1972) 167-169 MR0309106

[2] J M Boardman, R M Vogt, Homotopy invariant algebraic structures on topological spaces, Lecture Notes in Math. 347, Springer, Berlin (1973) MR0420609

[3] T tom Dieck, Topologie, second edition, de Gruyter Textbook, Walter de Gruyter \& Co., Berlin (2000) MR1150244

[4] T tom Dieck, K H Kamps, D Puppe, Homotopietheorie, Lecture Notes in Math. 157, Springer, Berlin (1970) MR0407833 
[5] A Dold, Partitions of unity in the theory of fibrations, Ann. of Math. (2) 78 (1963) 223-255 MR0155330

[6] E Dror Farjoun, Cellular spaces, null spaces and homotopy localization, Lecture Notes in Math. 1622, Springer, Berlin (1996) MR1392221

[7] E Dyer, S Eilenberg, An adjunction theorem for locally equiconnected spaces, Pacific J. Math. 41 (1972) 669-685 MR0319143

[8] E E Floyd, W J Floyd, Actions of classical small categories, Distributed manuscript (1990)

[9] M Mather, Pull-backs in homotopy theory, Canad. J. Math. 28 (1976) 225-263 MR0402694

[10] J P May, The geometry of iterated loop spaces, Lectures Notes in Math. 271, Springer, Berlin (1972) MR0420610

[11] J P May, $E_{\infty}$ spaces, group completions, and permutative categories, from: "New developments in topology (Proc. Sympos. Algebraic Topology, Oxford, 1972)", (G Segal, editor), London Math. Soc. Lecture Note Ser. 11, Cambridge Univ. Press, London (1974) 61-93 MR0339152

[12] HD Meiwes, Approximation von $\Omega^{n} \Sigma^{n} X$ bis auf Homotopie, $\mathrm{PhD}$ thesis, University of Heidelberg (1981)

[13] H D Meiwes, Private communication (1983)

[14] D Puppe, Some well known weak homotopy equivalences are genuine homotopy equivalences, from: "Symposia Mathematica, Vol. V (INDAM, Rome, 1969/70)", Academic Press, London (1971) 363-374 MR0303528

[15] V Puppe, A remark on “homotopy fibrations", Manuscripta Math. 12 (1974) 113-120 MR0365556

[16] G Segal, Categories and cohomology theories, Topology 13 (1974) 293-312 MR0353298

[17] J Smrekar, CW type of inverse limits and function spaces arXiv:0708.2838v1

[18] R W Thomason, Uniqueness of delooping machines, Duke Math. J. 46 (1979) 217-252 MR534053

[19] R M Vogt, Commuting homotopy limits, Math. Z. 153 (1977) 59-82 MR0451237

An der Tentenburg 7, D-49074 Osnabrück, Germany

Universität Osnabrück, Fachbereich Mathematik/Informatik

Albrechtstr 28a, 49069 Osnabrück, Germany

eschwamb@arcor.de, rvogt@uos.de

http://www.mathematik. uni-osnabrueck.de/staff/phpages/vogtr.rdf .shtml

Received: 31 October $2008 \quad$ Revised: 3 April 2009 